\title{
AUFSÄTZE
}

\section{Bundestagswahl 2009: Ein Wechsel auf Raten}

\author{
Richard Hilmer
}

\section{Das Wahlergebnis}

Die Bundestagswahl 2009 stellt in mehrfacher Hinsicht eine Zäsur in der deutschen Wahlgeschichte dar. Nie gab es mehr Volatilität im Wahlverhalten und nie mehr Veränderungen in der Parteienlandschaft. Am Ende stand aber paradoxerweise ein Ergebnis mit einer bemerkenswert stabilen Regierungsmehrheit und einer altvertrauten Regierungskonstellation: eine Koalition aus CDU/CSU und FDP. Anders als 2005 sorgten die Wähler für klare Verhältnisse, denn sie verhalfen Union und Freidemokraten zu einer deutlichen Mandatsmehrheit von 332 gegenüber 290 Sitzen für SPD, Linke und Grüne. Sichergestellt wurde dieses Endresultat durch das beste Ergebnis, das die FDP jemals bei einer Bundestagswahl erzielte, und durch 24 Überhangmandate für die Union, mehr als jemals zuvor bei einer Bundestagswahl. Es wird zu klären sein, warum die Wähler diesmal Schwarz-Gelb zu der Mehrheit verhalfen, die viele schon 2002 erhofft und die 2005 die meisten erwartet hatten. Damals stimmten viele unionsgeneigte Wähler im entscheidenden Moment anders, weil sie Schwarz-Gelb eine „soziale Unwucht unterstellten, die sich vor allem an dem von Paul Kirchhof propagierten Steuermodell festmachte"1. Sind derlei Befürchtungen zwischenzeitlich entkräftet worden, oder spielte das Thema Soziale Gerechtigkeit bei dieser Bundestagswahl eine eher untergeordnete Rolle?

Auf den ersten Blick hat einzig die FDP den schwarz-gelben Wahlsieg gesichert, denn nur sie legte zu - ebenso wie die Grünen und die Linke. Die Union verlor nach ihrem enttäuschenden Abschneiden vor vier Jahren ein weiteres Mal an Stimmen, wenn auch deutlich weniger als ihr Koalitionspartner SPD. Einbußen für Regierungsparteien sind eher Normalität, ungewöhnlich war jedoch deren Ausmaß. Einen vergleichbaren Swing - die Verluste der beiden Regierungsparteien summierten sich auf 12,6 Prozentpunkte, die Gewinne der drei Oppositionsparteien auf 10,6 Punkte - hat es bei Bundestagswahlen seit 1953 nicht mehr gegeben. Der Erklärung bedarf insbesondere die desaströse Niederlage der SPD. Sie verlor innerhalb nur einer Wahlperiode über sechs Millionen Stimmen, im Vergleich zu ihrem Wahlsieg bei der Bundestagswahl 1998 sogar die Hälfte ihrer Wählerschaft. Mit einem Zweitstimmenanteil von 23 Prozent blieb sie deutlich hinter ihrem bislang schlechtesten Ergebnis von 28,8 Prozent aus dem Jahre 1953 zurück.

1 Vgl. hierzu Richard Hilmer, Bundestagswahl 2002: eine zweite Chance für Rot-Grün, in: ZParl, 34. Jg. (2003), H. 1, S. 187 - 219 sowie Richard Hilmer / Rita Müller-Hilmer, Die Bundestagswahl vom 18. September 2005: Votum für einen Wechsel in Kontinuität, in: ZParl, 37. Jg. (2006), H. 1, S. $183-218$. 


\begin{tabular}{|l|c|c|c|c|c|}
\hline Tabelle 1: & $\begin{array}{c}\text { Bundestagswablergebnisse } 1998 \text { bis } 2009 \text { im Vergleich } \\
\text { (Zweitstimmenanteile in Prozent) }\end{array}$ \\
\hline Partei & 1998 & 2002 & 2005 & 2009 & Diff. 09-98 \\
\hline CDU & 28,4 & 29,5 & 27,8 & 27,3 & $-1,1$ \\
CSU & 6,7 & 9,0 & 7,4 & 6,5 & $-0,2$ \\
SPD & 40,9 & 38,5 & 34,2 & 23,0 & $-17,9$ \\
FDP & 6,2 & 7,4 & 9,8 & 14,6 & $+8,4$ \\
Die Linke* & 5,1 & 4,0 & 8,7 & 11,9 & $+6,8$ \\
Grüne & 6,7 & 8,6 & 8,1 & 10,7 & $+4,0$ \\
Sonstige & 6,0 & 3,0 & 3,9 & 6,0 & 0,0 \\
\hline * 2005 = Linke.PDS, 2002 und 1998 = PDS. \\
Quelle: Bundeswahlleiter.
\end{tabular}

Zum zweiten Mal in Folge konnte die Union nicht von den Verlusten der SPD profitieren. Schmerzlich ist dies vor allem für die CSU, nicht nur weil sie etwas stärker verlor als ihre Schwesterpartei, sondern vor allem weil sie jetzt schon zum zweiten Mal hintereinander die schwächste der sechs im Bundestag vertretenen Parteien ist. Mit 27,3 und 6,5 Prozent erzielten CDU und CSU ebenfalls ihr jeweils schlechtestes Ergebnis bei Bundestagswahlen seit $1953^{2}$. Dies wurde allerdings dadurch etwas relativiert, dass die Christdemokraten dank der Überhangmandate gegenüber 200514 Parlamentssitze hinzugewannen und dass die Union mit ihrem erklärten Wunschpartner FDP wieder die Regierung stellt.

Der Prozess der „Dekonzentration des Parteiensystems“3, verbunden mit einem Bedeutungsverlust der beiden Volksparteien ${ }^{4}$, hat sich bei dieser Wahl deutlich beschleunigt. Entfielen 2002 immerhin 77 Prozent und 2005 noch knapp 70 Prozent der abgegebenen Stimmen auf Union und SPD5 , so waren es diesmal noch ganze 56,8 Prozent. Ein erheblicher Teil der Verluste der Sozialdemokraten, aber auch die der Union gehen auf das Konto derer, die diesmal der Wahl fern blieben. Mit den Zugewinnen der drei kleinen Oppositionsparteien hat sich das Fünf-Parteien-System, das sich nach der deutschen Vereinigung herausgebildet hat, verfestigt; gleichzeitig erodierte aber seine bipolare Struktur mit zwei starken Volksparteien als Gravitationszentren der beiden Lager. Die Bundestagswahl 2009 brachte den „kleinen“ Parteien eine bis dato kaum vorstellbare Stärke und politisch mehr Durchsetzungskraft. Bei Anteilen von jeweils über zehn Prozent hat auch die Fünf-Prozent-Sperrklausel für FDP, Linke und Grüne ihren existenziellen Schrecken verloren. Dies dürfte zumindest den Liberalen und den Grünen in ihrer Rolle als „Scharnierpartei“ zum jeweils anderen politischen Lager neue koalitionäre Spielräume verschaffen ${ }^{6}$.

2 Nur bei der ersten Bundestagswahl 1949 schnitten CDU und CSU mit 31 Prozent schlechter ab.

3 Vgl. Bernhard Weßels, Splitting sichert den Wechsel, in: WZB-Mitteilungen, H. 129 (Dezember 2009), S. 33 - 37, S. 34.

4 Zur Definition der Volksparteien und zu den Ursachen für deren Niedergang siehe auch Peter Lösche, Ende der Volksparteien, in: APuZ, B 51 (2009), S. 6 - 12.

5 Bis 1987 vereinten Union und SPD noch 87 Prozent, 1976 sogar 91,2 Prozent der Stimmen auf sich.

6 Vgl. Karl-Rudolf Korte, Neue Qualität des Parteienwettbewerbs im „Superwahljahr“, in: APuZ, B 38 (2009), S. $3-8$. 


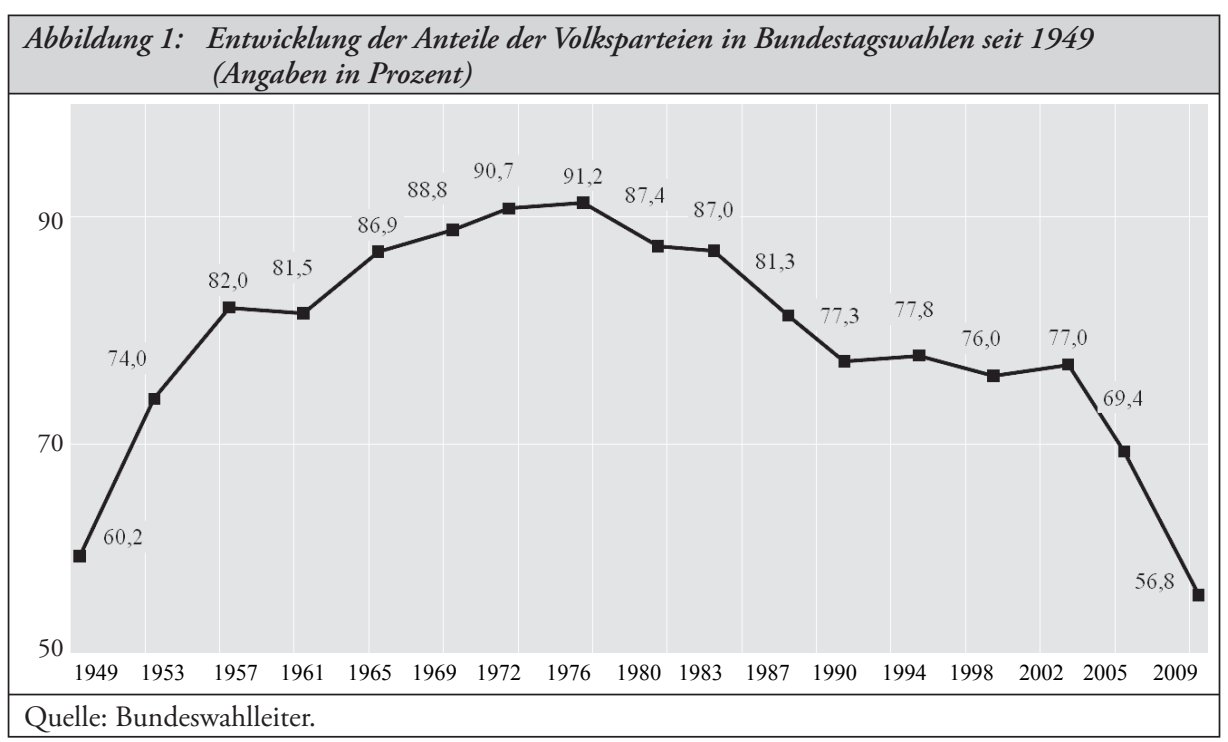

Ausgeblieben ist das mit der Wiederauflage der Großen Koalition befürchtete Erstarken rechtsradikaler Parteien, das bei der ersten Großen Koalition Ende der 1960er Jahre noch eingetreten war. Der Anteil kleinerer Parteien nahm zwar in der Summe zu, aber letztlich vermochte es keine der übrigen 21 angetretenen Parteien, auch nur in die Nähe der FünfProzent-Hürde zu kommen. Einzig die Piratenpartei sorgte im ersten Jahr ihres Bestehens mit einem Stimmenergebnis von zwei Prozent für ein Ausrufezeichen, zumal sie bei den männlichen Jungwählern sogar ein zweistelliges Ergebnis erzielte.

Diese Wahl geht mit einer ganzen Reihe weiterer Rekordmarken in die Annalen der deutschen Wahlgeschichte ein ${ }^{7}$, deren Auswirkungen weit über diese Wahlperiode hinausreichen dürften. Die Bundestagswahl 2009 brachte (1) die größten Kräfteverschiebungen zwischen den Parteien seit 1953 - Gewinne und Verluste der Parteien addieren sich auf 25,2 Punkte; (2) den größten Verlust, den je eine Partei zu beklagen hatte - minus 11,2 Prozentpunkte für die SPD und (3) mit 70,8 Prozent die mit Abstand niedrigste Beteiligungsrate bei einer Bundestagswahl - erstmals war die Zahl der Nichtwähler größer als die Zahl der Wähler der stärksten Partei ${ }^{8}$. Damit hat der Trend sinkender Wahlbeteiligung, der seit langem bei Landtags-, Kommunal- und vor allem bei Europawahlen zu beklagen war, mit voller Wucht auch die Bundesebene erreicht.

Neben der sinkenden Wahlbeteiligung und dem Bedeutungsverlust der Volksparteien bestätigt das Ergebnis der Bundestagswahl 2009 auch eine Reihe weiterer Trends:

Der Anteil der Wechselwähler hat mit einem Anteil von 31 Prozent eine neue Höchstmarke erreicht - bei den Bundestagswahlen 1998 und 2002 gab nur jeder vierte Wähler einer anderen Partei seine Zweitstimme als vier Jahre zuvor, 2005 waren es 28 Prozent.

7 „Bestes, höchstes, niedrigstes - das Wahlverhalten der Deutschen am 27. September lässt sich ... nur unter Zuhilfenahme einer bisher kaum für möglich gehaltenen Anzahl von Superlativen interpretieren." Daniel Deckers, Die Schere schließt sich, in: FAZ vom 29. September 2009, S. 2.

8 Gemessen an den Wahlberechtigten betrug der Anteil der Union 23,6 Prozent und der der SPD 16 Prozent, der der Nichtwähler aber 29,2 Prozent. 
Gestiegen ist ein weiteres Mal der Anteil der Briefwähler, begünstigt durch eine Lockerung der Voraussetzungen für diese ursprünglich als Ausnahme gewährte Form der Stimmabgabe. Er stieg kontinuierlich von 16,1 Prozent 1998 auf 18,0 im Jahre 2002, 18,7 in 2005 auf jetzt 21,4 Prozent. Diese Form der Wahlbeteiligung nehmen vor allem ältere Menschen sowie mobile Wähler mit höherem Bildungsabschluss wahr.

Auch der Anteil der Wähler, die ihre Erst- und Zweitstimmen unterschiedlichen Parteien gegeben haben, hat einen Rekordstand erreicht. Er stieg von 21 Prozent bei der Bundestagswahl 2002, über 24 Prozent 2005 auf jetzt 27 Prozent. Dies resultiert aus dem Stimmenzuwachs von FDP und Grünen, deren Wähler bevorzugt von der Möglichkeit des Stimmensplittings Gebrauch machen.

Parallel zur Bundestagswahl fanden am 27. September Landtagswahlen in SchleswigHolstein und Brandenburg statt. In Schleswig-Holstein unterschieden sich die auf beiden Wahlebenen erzielten Ergebnisse der Parteien nur unwesentlich voneinander ${ }^{9}$ - so wie es in der Vergangenheit bei zeitgleichen Bundes- und Landtagswahlen immer der Fall war. Ungewöhnlich war allerdings das Wahlverhalten der Brandenburger. Bei der Bundestagswahl straften sie wie überall die SPD deutlich ab, auf Landesebene bestätigten sie die Sozialdemokraten aber wieder als eindeutig stärkste Kraft. Am Ende entschieden sich auf Landesebene 33 Prozent für die SPD (+1,1 Prozentpunkte), auf Bundesebene aber nur 25,1 $(-10,7)^{10}$. Wähler scheinen heute nicht nur eher zum Wechsel bereit zu sein; bei zeitgleichen Wahlen wägen sie auch stärker das Für und Wider der Parteien auf Bundes- und Landesebene $\mathrm{ab}$.

\section{Rückblick auf die 16. Wahlperiode des Bundestages}

Das Wahlergebnis vom 18. September 2005 hätte rein arithmetisch neben einer Großen Koalition auch drei Dreierkoalitionen ermöglicht. Nachdem aber der FDP-Vorsitzende Guido Westerwelle schon am Wahlabend ein Zusammengehen mit den "Wahlverlierern" SPD und Grünen ebenso klar ausgeschlossen hatte wie umgekehrt die Grünen die aus ihrer Sicht nur vordergründig reizvolle "Jamaika-Koalition“, blieb am Ende nur eine Große Koalition (ein rot-rot-grünes Bündnis hätte zwar ebenfalls über eine knappe Mehrheit verfügt, wurde aber von keiner der drei Parteien ernsthaft in Erwägung gezogen). Union und SPD hatten zwar beide deutlich verloren, erreichten aber mit zusammen 448 von 614 Sitzen fast eine Drei-Viertel-Mehrheit. Am 18. November 2005 unterzeichneten die CDUVorsitzende und designierte Kanzlerin Angela Merkel sowie der SPD-Vorsitzende und designierte Arbeitsminister und Vizekanzler Franz Müntefering den Vertrag zur Bildung der zweiten Großen Koalition in der Geschichte der Bundesrepublik. Die Ressortverteilung gab das Kräfteverhältnis zweier fast gleichstarker Partner wieder und orientierte sich an klassischen Verteilungsmustern: Die Union stellte neben der Kanzlerin die Minister für Inneres und Wirtschaft, die SPD dafür den Außen-, Justiz- und Finanzminister. Bemerkenswerter

9 So war es auch diesmal in Schleswig-Holstein der Fall, wo die maximale Differenz zwischen Landes- und Bundesergebnis 1,9 Punkte betrug: die Linke kam im Bund auf 7,9, im Land dagegen nur auf 6,0 Prozent.

10 Nutznießer der bundespolitischen Schwäche der SPD war vor allem die brandenburgische CDU, die bei der Bundestagswahl um 3,8 Punkte besser abschnitt als bei der Landtagswahl. 
war, dass die Sozialdemokraten die beiden in der Regel undankbaren Ressorts Arbeit und Gesundheit übernahmen und der Union dafür die beiden Zukunftsressorts Bildung und Familie überließen. Für das Familienministerium fand die CDU in Ursula von der Leyen eine Idealbesetzung, was sich mit Blick auf die notwendig gewordene gesellschaftspolitische Neupositionierung der Union als Glücksgriff erwies.

Als eine der ersten Maßnahmen beschloss die Regierung Merkel / Müntefering die Anhebung der Mehrwertsteuer von 16 auf 19 Prozent, womit sie sich gleich zu Beginn ihrer Amtsperiode den Vorwurf des Wahlbetrugs einhandelte. Die Union hatte zwar im Wahlkampf eine Erhöhung der Mehrwertsteuer angekündigt - allerdings nur um zwei Punkte; diese war seinerzeit von der SPD heftig bekämpft worden („Merkelsteuer“). Die Mehreinnahmen sollten zum einen der Reduzierung der Neuverschuldung dienen, zum anderen der Finanzierung ambitionierter Reformvorhaben wie der Neugestaltung des Gesundheitswesens, der Föderalismusreform sowie von Maßnahmen zur Bekämpfung der Arbeitslosigkeit - schließlich verstand sich die CDU/CSU-SPD-Regierung nicht als Not-, sondern als Reformbündnis. So war auch die Wahrnehmung in der Bevölkerung. Eine knappe Mehrheit der Bürger war damals überzeugt, dass ein Bündnis aus Union und SPD die beste Regierungskonstellation sei, um das Land wieder voranzubringen ${ }^{11}$. Die erste Bilanz der Wähler fiel auch entsprechend positiv für die Regierung aus ${ }^{12}$.

Diese positive Grundstimmung kam - wie fast durchgängig während der Amtsperiode der Großen Koalition - in erster Linie der Union zugute. Sie wurde als die treibende Kraft in der Regierung angesehen und konnte sich in den Umfragen mit klarem Vorsprung von ihrem Bündnispartner absetzen. Der schwache Start der SPD war nicht zuletzt Folge innerparteilicher Querelen, die in Münteferings Rücktritt vom Amt des Parteivorsitzenden mündeten. Für die SPD kam dieses Umfragetief zu einem besonders ungünstigen Zeitpunkt, denn im März 2006 standen Landtagswahlen in Rheinland-Pfalz, Baden-Württemberg und Sachsen-Anhalt an. In Rheinland-Pfalz ging es dabei um den Machterhalt des einzig verbliebenen SPD-Ministerpräsidenten in einem westdeutschen Flächenland. Für die schlechten Umfragewerte wurden insbesondere der Arbeitsminister Franz Müntefering und sein Nachfolger im Parteivorsitz Matthias Platzeck verantwortlich gemacht, wenn auch aus unterschiedlichen Gründen. Müntefering hatte innerhalb der eigenen Partei Erstaunen bis Fassungslosigkeit mit seinem Vorstoß ausgelöst, die Rente mit 67 bereits früher als geplant einzuführen. Dabei handelte es sich eigentlich um ein Wunschprojekt der Union, das von Müntefering mit großem Einsatz umgesetzt wurde. Viele SPD-Anhänger werteten das jedoch als eine faktische Rentenkürzung. Platzeck, der noch vier Monate zuvor auf dem Karlsruher Parteitag mit einem überragenden Ergebnis von 99,4 Prozent der Stimmen von den Delegierten zum neuen Parteivorsitzenden gewählt worden war, sah sich innerparteilich schon früh dem Vorwurf der Führungsschwäche ausgesetzt ${ }^{13}$. Die Landtagswahlen im selben Monat verliefen für die SPD mit Ausnahme der massiven Verluste in Baden-Württemberg gleichwohl relativ glimpflich. In Rheinland-Pfalz führten die moderaten Zugewinne für die Sozialdemokraten und das schwache Abschneiden der Konkurrenz sogar zum

11 Vgl. Infratest dimap, ARD-DeutschlandTrend vom Oktober 2005, S. 3.

12 Vgl. Infratest dimap, ARD-DeutschlandTrend vom Dezember 2005, S. 2.

13 Diese Auseinandersetzungen schlugen sich auch in sinkenden Sympathiewerten der beiden sozialdemokratischen Protagonisten nieder. Vgl. Infratest dimap, ARD-DeutschlandTrend vom März 2006, S. 6. 
Gewinn der absoluten Mehrheit. Als Matthias Platzeck wenige Wochen später auf Grund gesundheitlicher Probleme sein Amt als SPD-Vorsitzender aufgab, einigte sich die Parteispitze rasch auf den rheinland-pfälzischen Wahlsieger und bisherigen stellvertretenden Vorsitzenden Kurt Beck als Nachfolger.

Auch für die Union war der Zenit in den Umfragewerten bereits im März 2006 erreicht. Das restliche erste Jahr verlief insbesondere für die CDU weitgehend glücklos. Bei allen Landtagswahlen musste sie Einbußen hinnehmen, und auf Bundesebene verlor die Partei der Kanzlerin ebenfalls an Zustimmung. Das dominierende Thema dieser Monate war die Reform des Gesundheitssystems - eine Diskussion, bei der sich die SPD im Laufe der Verhandlungen eine Kompetenzführerschaft gegenüber dem Koalitionspartner erarbeiten konnte und es zu teilweise heftigen Auseinandersetzungen innerhalb von CDU und CSU kam. Auf Widerstand stieß die Kanzlerin insbesondere bei den Ministerpräsidenten der unionsgeführten Länder. So musste Merkel mit der SPD mühsam ausgehandelte Kompromisse wie die geplanten Steuererhöhungen zur Finanzierung der Gesundheitsreform auf Druck aus der eigenen Partei zurücknehmen und die vereinbarte „Überforderungsklausel“ zur Begrenzung der Zusatzprämie auf maximal ein Prozent des Einkommens gegen starken Widerstand durchdrücken. Die Folge war ein Bild völliger Zerstrittenheit der Partei in der Wahrnehmung der Bevölkerung ${ }^{14}$ mit der Konsequenz, dass die Union im Oktober 2006 mit nur noch 30 Prozent der Stimmen erstmals - und auch letztmals - hinter die SPD zurückfiel ${ }^{15}$. Als die Landeschefs von CDU und CSU mit Blick auf anstehende Wahlen im eigenen Land ihre Angriffe auf die Bundesregierung einstellten, verbesserte sich das Ansehen der Union rasch wieder.

In den folgenden zwei Jahren gelang es der Regierung zunehmend, Vertrauen in der Bevölkerung zu gewinnen. Vor dem Hintergrund guter Wirtschaftsdaten, sinkender Arbeitslosigkeit und Erfolgen bei der Konsolidierung des Haushaltes erreichte die Große Koalition im Oktober 2007 ihre beste Bewertung während der 16. Wahlperiode. Damals waren 44 Prozent der Deutschen mit der Arbeit ihrer Regierung zufrieden. Erneut profitierte davon aber ausschließlich die CDU/CSU, die sich mit einem Stimmenanteil von 40 Prozent und 13 Punkten Vorsprung klar gegenüber ihrem Bündnispartner absetzen konnte ${ }^{16}$. Grund dafür war nicht nur die gelungene Profilierung der Union auf der personellen, sondern auch auf der sachpolitischen Ebene. Ihr gelang es über weite Strecken sogar, auf denjenigen Feldern einen Kompetenzvorsprung gegenüber den Sozialdemokraten zu erreichen, auf denen diese den Ressortchef stellten. Dies galt für die Haushalts- und Finanzpolitik genauso wie für die Arbeitsmarkt- und Außenpolitik.

Innerhalb der SPD flammten zu diesem Zeitpunkt erneut innerparteiliche Diskussionen auf um ein politisches Erbe der rot-grünen Vorgängerregierung. Beck setzte im SPD-Parteipräsidium die Verlängerung der Bezugsdauer des Arbeitslosengeldes I gegen den Widerstand von Müntefering und Finanzminister Peer Steinbrück durch, die darin eine Abkehr von den Reformen der Agenda 2010 sahen. In den folgenden Monaten geriet der Parteivorsitzende nicht zuletzt auch wegen der Entwicklungen in Hessen immer stärker in Bedrängnis. Nach der hessischen Landtagswahl im Januar 2008, die die CDU die Mehrheit kostete, verkündete die Landesvorsitzende der SPD, Andrea Ypsilanti, entgegen ihren Erklärungen vor der

14 Vgl. Infratest dimap, ARD-DeutschlandTrend vom Oktober 2006, S. 4.

15 Vgl. ebenda, S. 8.

16 Vgl. Infratest dimap, ARD-DeutschlandTrend vom Oktober 2007, S. 13. 
Wahl, mit der Linkspartei über die Duldung einer rot-grünen Minderheitsregierung verhandeln zu wollen. Dies brachte ihr seitens des politischen Gegners, aber auch seitens großer Teile der Öffentlichkeit den Vorwurf des Wortbruchs ein. Als ihr Plan am Widerstand aus den eigenen Reihen zunächst scheiterte, startete sie im Juli einen erneuten Anlauf, der aber ebenso misslang. In innerparteiliche Kritik geriet auch Beck, der unmittelbar vor der Wahl in Hamburg zum Entsetzen der dortigen SPD den hessischen Genossen beim ersten Versuch freie Hand ließ und beim zweiten Anlauf zwar davor warnte - „man läuft nicht zweimal mit demselben Kopf gegen dieselbe Wand“ -, ihn aber nicht verhinderte. Bei der SPD-Klausur Anfang September 2008 überschlugen sich dann die Ereignisse: Beck legte den Parteivorsitz nieder und ließ Frank-Walter Steinmeier den Vortritt in Sachen Kanzlerkandidatur. Noch am selben Tag schlug Steinmeier Franz Müntefering als neuen Parteivorsitzenden vor. Es war der vierte Wechsel an der Spitze der Partei innerhalb von fünf Jahren.

Die 16. Wahlperiode des Deutschen Bundestages ist auch mit einem weiteren Bedeutungsschwund der Volksparteien in den Landesparlamenten verbunden. Die Unionsparteien konnten ihre beherrschende Stellung in den Ländern, die sie zu Zeiten der rot-grünen Koalition aufbauten, nicht halten und verloren in sechs Bundesländern ihre absolute Mehrheit. Davon konnte jedoch die SPD nur bedingt profitieren. Neuen Regierungsbeteiligungen in Sachsen-Anhalt und Thüringen stehen Machtverluste in Sachsen und Schleswig-Holstein gegenüber, wo die SPD jeweils von der FDP als Regierungspartner der CDU abgelöst wurde. Nicht nur dort waren die Oppositionsparteien die Gewinner. Die FDP musste nur bei den Wahlen 2006 in Sachsen-Anhalt und in Berlin Verluste hinnehmen, bei allen anderen Wahlen konnte sie ihren Stimmenanteil zum Teil deutlich ausbauen oder zumindest halten. Bei der Wahl zum Europäischen Parlament im Juni 2009 gelang ihr mit einem Zuwachs von knapp fünf Punkten ebenfalls ein beachtlicher Erfolg. Auch die Grünen konnten bei den Landtagswahlen von 2005 bis 2009 überwiegend Gewinne verbuchen. Am deutlichsten fielen diese bei der Neuwahl in Hessen im Januar 2009 aus, wo die Grünen von den dramatischen Verlusten der SPD profitieren konnten. Die neu gegründete Linkspartei, die im Juni 2007 aus der Fusion von Linke.PDS und WASG entstanden war, schaffte es in der vergangenen Wahlperiode, auch im Westen Deutschlands als politische Kraft Fuß zu fassen. Bei allen nach 2006 in westdeutschen Bundesländern stattfindenden Wahlen gelang der Linken der Einzug ins Parlament - von Bayern abgesehen. Am eindrucksvollsten fiel der Erfolg im Saarland im August 2009 aus, wo sie aus dem Stand drittstärkste Partei wurde und über ein Fünftel der Wählerschaft binden konnte. Diese Entwicklungen blieben nicht ohne Konsequenzen für die Mehrheitsverhältnisse im Bundesrat. Mit der vorgezogenen Neuwahl in Hessen, die einer schwarz-gelben Regierungskoalition zur Mehrheit verhalf, büßte die Große Koalition im Januar 2009 ihre Mehrheit in der Länderkammer ein.

Das letzte Jahr der schwarz-roten Bundesregierung stand ganz im Zeichen der Finanzund Wirtschaftskrise und dem Bemühen der Bundesregierung, die deutschen Sparer zu beruhigen und die Folgen der Krise einzudämmen. Das erste Konjunkturpaket, das die Bundesregierung Anfang November 2008 zu diesem Zweck auf den Weg brachte und das immerhin die höchste Nettoneuverschuldung seit Bestehen der Bundesrepublik zur Folge hatte, stieß in der Bevölkerung auf viel Zustimmung. ${ }^{17}$ Die guten Noten, die die Deutschen der Regierung für ihr Krisenmanagement ausstellten, nutzten einmal mehr aus- 
schließlich der Union, während die SPD weiter im Stimmungstief verharrte. Auch von ihr initiierte und von der Öffentlichkeit begrüßte Maßnahmen, wie etwa die Ausweitung des Kurzarbeitergeldes, wurden ihr nicht gutgeschrieben. Die Ausgangsposition der Sozialdemokraten vor der Wahl wurde zudem durch die gescheiterte Regierungsübernahme in Hessen belastet; Diskussionen um den künftigen Kurs der Bundesbahn sowie in der Schlussphase die „Dienstwagenaffäre“, die in themenarmer Zeit lange die Schlagzeilen beherrschte, kamen hinzu. Mit der anhaltenden Wirtschafts- und Finanzkrise nahm jedoch auch das Vertrauen der Bürger in die Krisenpolitik der Bundesregierung ab. Entscheidungen wie die wiederholt geforderten Finanzhilfen für angeschlagene Banken stießen bereits im März 2009 bei einer Mehrheit auf Ablehnung ${ }^{18}$, und die geplanten staatlichen Hilfen für Opel wurden von Beginn an sehr skeptisch bewertet. Dadurch wuchs auch der Druck auf die Union, die auf Grund von Kompetenzverlusten im Bereich der Wirtschaftspolitik einen Verlust an Wählerstimmen insbesondere an die FDP befürchten musste. Diese Kompetenzverschiebung ging einher mit schlechten Noten für den CSU-Wirtschaftsminister Michael Glos, der nach anhaltenden innerparteilichen Grabenkämpfen mit seinem neuen Parteivorsitzenden Horst Seehofer die Konsequenzen zog und zurücktrat - der einzige Rücktritt in der ersten Regierungsperiode Merkels. Als Nachfolger wurde CSU-Generalsekretär KarlTheodor zu Guttenberg bestellt. In der Auseinandersetzung um die Staatshilfe für Opel vermochte er sich schnell als Kämpfer für eine marktwirtschaftliche Ordnungspolitik zu profilieren und der Wirtschaftskompetenz der Union wieder ein Gesicht und mehr Profil zu geben. Die Wirtschafts- und Finanzkrise und daraus resultierende Schieflagen renommierter Unternehmen (Opel, Schäffler, Arcandor) beherrschten die politische Agenda und drängten das übrige politische Geschehen in den Hintergrund - mit einer einzigen Ausnahme: Als drei Wochen vor der Wahl ein deutscher Oberst den Befehl zur Zerstörung von zwei von den Taliban entführten Tanklastzügen bei Kundus gab, stand für einige Tage der Afghanistan-Konflikt im Fokus der Öffentlichkeit. So schnell er auftauchte, so schnell verschwand er aber auch wieder von der Tagesordnung. Auf den Wahlausgang hatte dieser Vorfall kaum Auswirkung, die Außen- und Sicherheitspolitik spielte jedenfalls ausweislich der ARD-Wahltagsbefragung eine deutlich geringere Rolle als $2005^{19}$.

\section{Die Ausgangslage und der Wahlkampf der Parteien}

\section{1. $\mathrm{CDU} / \mathrm{CSU}$}

Wer glaubte, vier Jahre Große Koalition seien gleichbedeutend mit vier Jahren permanenten Wahlkampfs, sah sich früh getäuscht. Das Gegenteil war der Fall, denn beide Partner gingen während der gesamten Wahlperiode weitgehend pfleglich miteinander um. Dazu trug anfangs das gute Verhältnis zwischen Merkel und Müntefering bei; aber auch nachdem sich Müntefering, frei von jeglichen Regierungsverpflichtungen, zunehmend kritisch zu Merkel äußerte, blieb der Ton innerhalb der Regierung konziliant. Auch bei den Landtags-

18 Vgl. Infratest dimap, ARD-DeutschlandTrend vom März 2009, S. 4.

19 Vgl. Infratest dimap, Wahlreport Bundestagswahl 2009, Berlin 2009, S. 62. Seine Sprengkraft für die Bundesregierung entfaltete dieser Angriff erst deutlich nach der Wahl, als Ex-Verteidigungsminister Franz JosefJung, mittlerweile Arbeitsminister in der zweiten Merkel-Regierung, deshalb zurücktreten musste und sein Nachfolger $z u$ Guttenberg in erhebliche Turbulenzen geriet. 
wahlen während der letzten vier Jahre wirkte sich die Große Koalition eher sedativ auf die Wahlkämpfe aus, galt es doch Rücksicht zu nehmen auf den Koalitionspartner in Berlin. Der Ausbruch der Finanz- und Wirtschaftskrise erforderte eine verstärkte Kooperation, denn jetzt stand doch die gemeinsame Bewältigung der Krise im Vordergrund. Für Wahlkampf blieb daher bis zum Sommer 2009 kaum Raum.

Dies kam der Union sehr gelegen, weil sie unbedingt einen Richtungswahlkampf wie 2005 vermeiden wollte, um möglicherweise immer noch lebendige Vorbehalte gegenüber einer „neoliberalen“ Koalition von Union und FDP erst gar nicht aufkommen zu lassen. Der sehr zurückhaltende Wahlkampf entsprach auch dem Führungsstil von Angela Merkel, die Zuspitzungen politischer Themen und Polarisierungen eher zu vermeiden suchte. In der Wirtschafts- und Finanzkrise konnte sie sich als Krisenmanagerin bewähren, zudem kam die höhere Kompetenzzuschreibung an die Union in wirtschaftspolitischen Fragen stärker zum Tragen. Mit der Formel „Regieren statt Wahlkampf“ gelang es ihr, den Kanzlerinnenbonus bis zum Schluss voll auszuspielen, während ihr Herausforderer Probleme hatte, sich in der extrem kurzen Endphase des Wahlkampfs als Kanzlerkandidat zu profilieren. Die Union ging in diesen „Nicht-Wahlkampf“ mit einer denkbar einfachen, aber sehr erfolgreichen Strategie: deren erstes Credo lautete „Merkel muss Kanzlerin bleiben“. Der Grundstein dafür wurde schon in der Frühphase der Großen Koalition gelegt, als die Kanzlerin ihren Führungsanspruch innerhalb der Union gegen die mächtigen Ministerpräsidenten durchsetzte und in der Folgezeit festigte. Für Merkel brachte dies den Spielraum, die CDU in einigen Themenfeldern etwas nach links zu rücken, so etwa in der Familienpolitik, aber auch in ausgewählten Feldern der Arbeitsmarktpolitik. Dort ließ sie es sogar - sehr zum Unwillen ihres SPD-Partners Müntefering - geschehen, dass führende Unionspolitiker (allen voran Jürgen Rüttgers) die SPD bei Hartz IV links überholten. Generell war die Kanzlerin bemüht, in Sachen sozialer Gerechtigkeit keine allzu großen Widersprüche zur SPD aufkommen zu lassen. Selbst zur Einführung von branchenspezifischen Mindestlöhnen erklärte sie sich zu weitgehenden Kompromissen bereit, den erwartbaren Ärger in den eigenen Reihen in Kauf nehmend. Zur Verstaatlichung der Hypo Real Estate als Reaktion auf deren selbstverschuldete Krise war es dann nur noch ein kleiner und konsequenter Schritt. Mit Blick auf den Wahlkampf immunisierte sie sich damit letztlich erfolgreich gegen Angriffe seitens der SPD, die Union stünde für soziale Kälte.

Damit ebnete sie den Weg für die Wunschkoalition mit der FDP. Diese Machtoption hatte zudem den Vorteil, den gerade in Krisenzeiten starken Wunsch vieler Wähler nach Kontinuität - in der Person der Kanzlerin - zu verbinden mit der nach vier Jahren Großer Koalition in der eigenen Anhängerschaft immer lauter werdenden Forderung nach einem Wechsel des Koalitionspartners. Im Wahlkampf adaptierte die Union Barack Obamas Wahlslogan „Yes we can“ in „Wir haben die Kraft" und setzte fast ausschließlich auf die Kanzlerin. Selbst weit über die CDU/CSU-Klientel hinaus beliebte Kabinettsmitglieder wie von der Leyen und zu Guttenberg, oder der für konservative Wähler wichtige Wolfgang Schäuble spielten im Unionswahlkampf kaum eine Rolle - sieht man einmal von der kurzen Plakatierung aller Kabinettsmitglieder einige Wochen vor der Wahl ab. Dies brachte der CDU/CSU vor der Wahl in den Medien den Vorwurf ein, einen „völlig sinnfreien“20 Wahlkampf zu bestreiten, und nach der Wahl machten einige Unionspolitiker Merkels 
Wahlkampf ${ }^{21}$ und ihren „präsidialen Stil“ für das „schlechteste Ergebnis nach 1949“ verantwortlich ${ }^{22}$.

Die CSU verzichtete weitgehend auf eine eigenständige Kampagne und stellte ebenfalls Merkel in den Mittelpunkt. Ihr „Shootingstar“ $z u$ Guttenberg wurde in Bayern jedoch weit intensiver plakatiert als in den anderen Bundesländern und nahm im Wahlkampf eine gleichbedeutende Stelle ein wie der CSU-Parteichef. Anders als Merkel versuchte Seehofer aber, sich im Wahlkampf vor allem durch Attacken gegen Guido Westerwelle von der FDP abzusetzen, was ihm manche innerparteiliche Kritik einbrachte und letztlich die Abwanderung vieler CSU-Wähler an die FDP nicht verhindern konnte.

\subsection{SPD}

Die SPD hatte von allen Parteien die komplizierteste Aufgabe zu bewältigen, denn ihr Wahlkampf war mit drei schweren Hypotheken belastet: unübersehbare Verschleißerscheinungen nach elf Jahren Regierungsbeteiligung, personelle Diskontinuität an der Spitze und der Mangel an einer glaubhaften Machtoption.

2005 war die SPD nach sieben Jahren Rot-Grün von der Kanzlerpartei zum Juniorpartner der Union degradiert worden. Schon am Wahlabend war deutlich geworden, dass die Partei dies nicht als Quittung für eine nicht mehr mehrheitsfähige Politik verstand, sondern eher als Auftrag für deren Fortführung. Sichtbarster Ausdruck dieser strategischen Positionierung innerhalb der Großen Koalition war die lange Verweigerung jeglicher Modifikationen bei Hartz IV. Dabei wurde übersehen, dass die breite Ablehnung einiger als ungerecht empfundener Aspekte von Hartz IV die gesamte Agendapolitik Gerhard Schröders zu diskreditieren drohte. Die gegen eigene Wahlversprechen vorgenommene Mehrwertsteuererhöhung sowie die kommunikativ ähnlich brachial wie die Agendapolitik durchgesetzte Rente mit 67 verstärkte bei vielen SPD-Wählern den Eindruck, die Sozialdemokratie habe die Lebenswirklichkeit der Bürger aus den Augen verloren. Dieser Konstruktionsfehler zog sich durch die gesamte Regierungsperiode und hatte zur Konsequenz, dass der Großteil der SPD-Wähler - im Gegensatz zu den Unionsanhängern - durchweg unzufrieden war mit der Großen Koalition. Sie erlebten die SPD in erster Linie als Exekutor einer unionsgeprägten Politik. Selbst dort, wo ur-sozialdemokratische Positionen durchgesetzt werden konnten (Mindestlohn, Verstaatlichung von Banken, Kurzarbeitergeld), musste die Partei diese Erfolge mit der Kanzlerin teilen. Versuche, die SPD innerhalb der Koalition neu zu positionieren, scheiterten meist schon in den ersten Ansätzen, so etwa Ende 2006 der Versuch des neuen Parteivorsitzenden Beck, sich an die Spitze einer gesellschaftspolitischen Debatte über Armut in Deutschland und über zentrifugale Kräfte in der bundesdeutschen Gesellschaft ${ }^{23}$ zu setzen. Schließlich wurde auch die mit der Bankenkrise verbundene Chance

21 Vgl. Axel Murswieck, Angela Merkel als Regierungschefin und Kanzlerkandidatin, in: APuZ, B 51 (2009), S. $26-32$.

22 So der Vorwurf mehrerer Landesfraktionsvorsitzender der CDU, öffentlichkeitswirksam publiziert in der Frankfurter Allgemeinen Sonntagszeitung. Vgl. Saskia Ludwig / Christean Wagner I Steffen Flath / Mike Mohring, Mehr Profil wagen!, in: FAS vom 10. Januar 2010, S. 9.

23 Angestoßen von der von TNS Infratest Sozialforschung im Auftrag der Friedrich-Ebert-Stiftung durchgeführten Studie „Gesellschaft im Reformprozess“. Deren Befunde bezüglich des Auseinanderdriftens gesellschaftlicher Gruppen wurde jüngst von einer in der FAZ vorgestellten Studie im 
vertan, sich in der Wirtschafts- und Sozialpolitik deutlicher vom Koalitionspartner abzusetzen.

Die zweite schwere Hypothek für den Wahlkampf der SPD war die personelle Ausgangssituation. Die häufigen Wechsel der Parteivorsitzenden - vier in vier Jahren - gaben den SPD-Anhängern nicht die notwendige Orientierung und verunsicherten die Mitglieder. Der letzte Wechsel von Beck zu Müntefering verlieh der Partei zwar wieder mehr Stabilität nach innen und brachte auch die lange offene Entscheidung über den Spitzenkandidaten für die Bundestagswahl. Die Doppelspitze Müntefering / Steinmeier bescherte der SPD aber eine latent offene Führungsfrage, was ihren Spitzenkandidaten gegenüber der Amtsinhaberin zusätzlich ins Hintertreffen geraten ließ ${ }^{24}$. Sein Wahlkampfauftakt missriet zudem, als er in der Debatte um öffentliche Unterstützungsmaßnahmen für Opel in die Defensive geriet und die SPD bei der EP-Wahl eine völlig unerwartete Niederlage hinnehmen musste. Das im Juni von Steinmeier vorgestellte erweiterte Wahlkampfteam bot zudem weniger Identifikationsmöglichkeiten für besonders volatile Wählergruppen (Junge, Frauen, Ostdeutsche) als die Union mit zu Guttenberg, von der Leyen und Merkel. In Steinmeier und Steinbrück hatte die SPD zwar ebenfalls Protagonisten mit höchsten Sympathiewerten, aber diese vermochten es nicht, die in elf Jahren Regierungsbeteiligung aufgebauten Vorbehalte gegenüber der SPD entscheidend abzubauen.

$\mathrm{Zu}$ alledem hatten die Sozialdemokraten auch keine realistische Machtperspektive. Die Parteispitze propagierte eine Koalition mit den Grünen und der FDP, die aber von der FDP unmissverständlich abgelehnt wurde. Die Ampel weckte zudem auch in der eigenen Anhängerschaft wenig Begeisterung. Die von ihr favorisierte Fortführung der Großen Koalition kam aus SPD-Sicht nicht in Frage, denn sie wäre angesichts des anhaltenden Stimmungstiefs der Partei als Kapitulation gegenüber dem Führungsanspruch der Union gedeutet worden. Die dritte rechnerisch mögliche Option, ein Zusammengehen mit der Linkspartei, wurde von den Sozialdemokraten immer wieder vehement zurückgewiesen.

Ob der SPD-Wahlkampf auch ohne diese Hürden an Fahrt gewonnen hätte, bleibt zweifelhaft, denn es fehlte bis zum Schluss an klaren und überzeugenden Aussagen. Aus Sicht der SPD-Wähler gab es zwar immer wieder hoffnungsvolle Ansätze, wie etwa die Rede des Spitzenkandidaten auf dem Wahlparteitag; so richtig in Schwung kam die Kampagne jedoch nie. Der im Juli von Steinmeier vorgelegte „Deutschlandplan“ verpuffte weitgehend wirkungslos, stattdessen bestimmte die „Dienstwagenaffäre“ die Agenda. Das Schlussmotto „Unser Land kann mehr“ erschloss sich den Wählern nicht so recht, da für viele unklar blieb, für welche Ziele die SPD eintreten würde. Es gelang auch keine thematische Zuspitzung; die in der Woche vor der Wahl plakatierte Warnung vor „sozialem Kahlschlag“ unter Schwarz-Gelb deckte sich nicht mit den Wahrnehmungen und der Stimmung der Wählerschaft. Ein fundamental kritisches Fazit zog Parteienforscher Joachim Raschke: „Keine Linie, keine Botschaft, kein Gesicht - diesmal war die SPD-Kampagne ein Nicht-Ereignis. "25

Wesentlichen bestätigt. Vgl. Franz Walter, Die Not der SPD begann viel früher, in: FAZ vom 11. Dezember 2009, S. 9.

24 In der Dimension „Führungsstärke“ fiel Steinmeier im Profilvergleich am deutlichsten hinter Merkel zurück.

25 Joachim Raschke, Zerfallsphase des Schröder-Zyklus. Die SPD 2005-2009, in: Christoph Egle I Reimut Zohlnhöfer (Hrsg.), Die Große Koalition 2005-2009, Wiesbaden 2010 (im Erscheinen). 


\subsection{FDP}

Den wohl stärksten Kontrast zur SPD-Kampagne bot der Wahlkampf der FDP. Sie hatte eine klare Führungsstruktur, eine stringente Programmatik und eine klare Machtperspektive. Guido Westerwelle, seit 2001 FDP-Vorsitzender und seit 2004 auch Fraktionsvorsitzender, wechselte nach seinem umstrittenen Ausflug in die Spaßpolitik im Wahlkampf 2002 rasch wieder in das seriöse Fach und verstand es in der Folgezeit, sein Ansehen in der Bevölkerung kontinuierlich zu verbessern. Das gute Abschneiden der Liberalen bei fast allen Landtagswahlen der zurückliegenden Jahre festigte seine innerparteiliche Position. Die Freidemokraten hatten auch eine klare Botschaft, die sie seit Jahren konsequent verkündeten: „Leistung muss sich wieder lohnen“; verbunden mit dem Versprechen, das Steuersystem zu vereinfachen und die Steuerbelastung zu senken, fand diese zunehmend auch bei enttäuschten Unions- und SPD-Wählern Anklang. Dieses zentrale Motto wurde im Laufe des Wahlkampfs noch ergänzt durch die Forderung nach mehr Investitionen in die Bildung sowie einer Stärkung der Bürgerrechte. Zudem hatte die FDP von allen Parteien die eindeutigste und wohl auch glaubhafteste Regierungsoption: Schwarz-Gelb. Dafür trat sie bereits 2005 ein, und davon ist sie auch ungeachtet der Werbungsversuche der SPD nie abgewichen. Diese Konstellation gewann an Realitätsgehalt noch dadurch, dass ein Flächenland nach dem anderen von einer schwarz-gelben Koalition regiert wurde. Selbst in Bayern zwangen die Wähler der CSU die Liberalen als Koalitionspartner auf und waren offenkundig zufrieden damit. Westerwelle wusste zudem die Angriffsflächen zu nutzen, die ihm die Große Koalition immer wieder bot - angefangen von der Erhöhung der Mehrwertsteuer auf 19 Prozent bis hin zu den in der Bevölkerung umstrittenen Unterstützungsmaßnahmen von Großkonzernen in der Krise. Den von der Wirtschaftspolitik der Großen Koalition enttäuschten Unionsanhängern bot sich die FDP erfolgreich als ordnungspolitische Alternative an. Ihr Wahlkampf mit seinem Slogan „die Mitte stärken“ mag unter Marketinggesichtspunkten keine Offenbarung gewesen sein, aber er war letztlich eminent erfolgreich und festigte die Position der FDP als klar drittstärkste Partei.

\subsection{Grüne}

Die Grünen, die bei der Bundestagswahl 2005 als Regierungspartei vergleichsweise glimpflich davongekommen waren, schärften in der Opposition wieder ihr umweltpolitisches Profil - und zwar mit Erfolg, denn sie werden heute noch stärker als früher mit dem Thema Umwelt- und Klimaschutz identifiziert. Und obwohl sich heute praktisch alle Parteien zu diesen Zielen bekennen, wird den Grünen in diesem Bereich ungebrochen die bei weitem größte Kompetenz zugesprochen - in Zeiten einer globalen Klimakrise ein gewichtiges Pfund. Auch die Zuspitzung ihrer wirtschaftspolitischen Aussagen auf ökologisch begründete Positionen dürfte den Grünen eher genutzt als geschadet haben. Die Partei verkraftete auch das Ausscheiden Joschka Fischers, ihres Frontmannes früherer Wahlkämpfe, ohne Probleme. Die Grünen verstanden es, die Spielräume, die sich ihnen als Oppositionspartei im Hinblick auf neue strategische Partnerschaften boten, zu ihren Gunsten zu nutzen. In der Hansestadt Bremen gingen sie nach erfolgreicher Wahl eine Koalition mit der SPD ein, in Hamburg entschieden sie sich für eine Koalition mit der CDU und damit für die erste schwarz-grüne Landesregierung. Geschadet hat den Grünen dieser Spagat nicht, sie blieben 
auch bei den nachfolgenden Landtagswahlen auf der Erfolgsspur. Ihre Delegierten fanden Gefallen an dieser Offenheit und lehnten die von der Parteiführung vorgeschlagene Wahlaussage zugunsten einer Ampel ab. Weder Jamaika noch ein Zusammengehen mit der Linkspartei wurden explizit ausgeschlossen. Als einziges Kriterium für die Auswahl möglicher Koalitionspartner ließen sie die optimale Durchsetzung grüner Forderungen gelten. Dies fand auch die Zustimmung ihrer Wähler, die stärker als Wähler anderer Parteien ihre Wahlentscheidung von politischen Inhalten abhängig machen. Personalpolitisch hielten die Grünen an der Trennung von Partei- und Fraktionsführung sowie an einer jeweiligen „doppelten Doppelspitze“ (Mann/Frau sowie links/realo) fest. Im Amt des Parteivorsitzenden löste Cem Özdemir Reinhard Bütikofer ab, der in das Europäische Parlament wechselte, und Jürgen Trittin übernahm den männlichen Part des Spitzenkandidaten und des späteren Fraktionsvorsitzenden, jeweils neben Renate Künast. Beide erwiesen sich als für die eigene Klientel überzeugende Protagonisten grün-ökologischer Politik. Im Wahlkampf dominierten ökologische Themen (Green Deal), die Forderung nach einem besseren Datenschutz sowie nach mehr Investitionen in die Bildung. Die Bekämpfung der Wirtschaftskrise wurde mit dem Klimaschutz verbunden: „Aus der Krise hilft nur Grün“. Der Lohn war das beste Wahlergebnis, das die Grünen jemals bei einer Bundestagswahl einfuhren.

\subsection{Linke}

Das gute gemeinsame Abschneiden bei der Bundestagswahl 2005 war für PDS und WASG das Startsignal für eine schnelle Zusammenführung beider Parteien. Vollzogen wurde dieser Schritt auf dem Gründungsparteitag im Juni 2007. Die Parteiführung wurde wie bereits die Fraktionsführung im Bundestag in die Hände einer Doppelspitze mit Ost-West-Besetzung gelegt. Für die alte PDS und damit den Osten übernahm noch einmal Lothar Bisky den Parteivorsitz, den WASG-Part übernahm wie schon in der Fraktion Oskar Lafontaine, der mit dieser Doppelfunktion zum starken Mann der Linken avancierte. Politisch war damit ein Linksrutsch verbunden, der als solcher auch von der Bevölkerung wahrgenommen wurde: Auf der Links-Rechts-Skala verorteten die Bundesbürger die Linkspartei nach dem Vereinigungsparteitag um 1,7 Punkte weiter links als vorher ${ }^{26}$. Die Linke verstand sich stärker noch als die PDS als Vertreterin der Interessen der unteren sozialen Schichten in Ost- und Westdeutschland. Damit war sie vor allem bei enttäuschten SPD-Anhängern erfolgreich, wobei Lafontaine als ehemaliger SPD-Vorsitzender im Westen eine wichtige Funktion als Türöffner zukam. Diese Neupositionierung hat der Linken nicht geschadet sondern eher genutzt, denn bei allen nachfolgenden Landtagswahlen im Westen, mit Ausnahme Bayerns, gelang ihr der Einzug ins Parlament, und auch in den neuen Bundesländern legte sie in den Wahlen durchweg zu - wiederum nur mit einer Ausnahme: in Berlin, dem einzigen Bundesland, in dem die PDS/Linke zu diesem Zeitpunkt an der Regierung beteiligt war. Nicht zuletzt wegen der dort verzeichneten deutlichen Verluste entbrannte ein heftiger Streit über die strategische Ausrichtung der Partei. Lafontaine, der sich an die Spitze der Kritiker einer Regierungsbeteiligung stellte, sah darin eine Folge einer allzu kompromissbereiten Politik, die im Widerspruch zum Kurs der Bundeslinken stehe. Die traditio- 
nell starken pragmatischen PDS-Kader in den ostdeutschen Landesverbänden hielten aber daran fest und strebten in der Folgezeit weitere Regierungsbeteiligungen an - in Thüringen ohne, in Brandenburg mit Erfolg.

Zu Beginn der Finanzkrise im Herbst 2008 deutete noch nichts auf ein Erstarken der Linkspartei hin. Zu diesem Zeitpunkt war eher praktische Krisenbewältigung angesagt, und die wurde zuallerletzt von der Linken erwartet. In dem Maße, in dem sich die Lage stabilisierte, legte die Linke in der Wählergunst wieder zu, erneut vor allem auf Kosten der SPD. Mit stark emotionalisierten Wahlparolen („Hartz IV muss weg“, „Reichtum für Alle“, „Deutsche Truppen raus aus Afghanistan“) gelang es ihr, ihre Wähler anzusprechen ${ }^{27}$ und letztlich auch besser zu mobilisieren als andere Parteien.

\subsection{TV-Duell(e)}

Höhepunkt des medialen Wahlkampfs war wie bei den zwei Wahlen zuvor das TV-Duell, das wiederum auf vier Kanälen ausgestrahlt wurde. Die Resonanz fiel diesmal aber deutlich geringer aus: Die Zahl der Zuschauer sank gegenüber 2005 von 20 auf 15 Millionen. Dies wurde in den Medien als Ergebnis des im Vergleich zu der Vorgängerwahl themenarmen und wenig mitreißenden Wahlkampfs interpretiert. Die gesunkene Sehbeteiligung war sichtbarster Ausdruck des geringen Interesses an diesem Wahlgang und gleichsam Menetekel für eine sinkende Wahlbeteiligung.

Die Auseinandersetzung zwischen Merkel und Steinmeier passte sich nahtlos in den bis dato wenig inspirierenden Wahlkampf ein und veranlasste taz und Bild zu dem selten einmütigen Gesamturteil „Yes, we gähn“28. Einzig das Thema Steuern brachte etwas Farbe in die Debatte. Hier konnte Steinmeier mit seinem Hinweis punkten, dass die Hoffnung von Union und FDP, die Steuererleichterungen durch den dadurch initiierten wirtschaftlichen Aufschwung gegenzufinanzieren, über Jahre ein Wirtschaftswachstum von über sieben Prozent voraussetzen würde - ein Wachstum, wie es die bundesdeutsche Wirtschaft allenfalls in den Zeiten des Wirtschaftswunders erlebt hatte. Insgesamt wirkte Steinmeier in der Diskussion etwas präsenter und besser präpariert, während Merkel erkennbar darum bemüht war, erst gar keine Gegensätze aufkommen zu lassen. Ihr Kalkül ging auf, weil sich beide immer wieder gegenseitig bestätigten, wie erfolgreich die Große Koalition letztlich agierte, und Steinmeier der Rollenwechsel vom Koalitionspartner zum Herausforderer nicht so recht gelingen mochte. Die Süddeutsche Zeitung titelte deshalb am nächsten Tag: „Duell gerät zum Duett“29. Aus den unmittelbar nach dem Duell durchgeführten Umfragen ging

27 Danach befragt, welche Partei den überzeugendsten Wahlkampf gemacht habe, setzten in einer Umfrage von Infratest dimap zwei Wochen vor der Wahl die eigene Partei auf Platz 1: die Anhänger der Linken zu 48 Prozent, die der Grünen zu 39 Prozent und die der FDP zu 35 Prozent. Am kritischsten gingen Anhänger von SPD und Union mit dem Wahlkampf ihrer Parteien zu Gericht: nur jeweils 30 Prozent setzten sie auf Platz 1.

28 Nikolas Blome, Yes we gähn!, bild.de vom 14. September 2009, http://www.bild.de/BILD/politik/2009/09/13/tv-duell-analyse/torloses-unentschieden-zwischen-merkel-und-steinmeier.html (Abruf am 09. Februar 2010); taz.de, Yes we gähn!, 14. September 2009, http://www.taz.de/1/ politik/deutschland/artikel/1/yes-we-gaehn (Abruf am 9. Februar 2010).

29 Sueddeutsche.de, Duell gerät zum Duett, 13. September 2009, http://www.sueddeutsche.de/politik/47/487452/text/ (Abruf am 8. Februar 2010). 
durchweg Steinmeier als knapper Sieger hervor und punktete vor allem bei unentschiedenen Wählern, was auch zu einem kurzfristigen Anstieg der Werte für die SPD führte. Diese Erfolge waren aber nicht zuletzt deshalb wenig nachhaltig, weil Themenfelder wie Bildung und Familie, die dieser Wählergruppe besonders wichtig waren, in dem Duell weder von den Moderatoren ${ }^{30}$ noch von den beiden Politikern in ihren Statements angesprochen wurden. Letztlich blieb das Duell ohne erkennbare Wirkung, denn Union und SPD schnitten bei der Wahl in etwa so ab, wie von den Umfragen vor dem Duell vorausgesagt.

Weit kontroverser zur Sache und wohl auch für die Zuschauer informativer ging es bei der anderntags in der ARD ausgestrahlten Diskussionsrunde der drei Oppositionsführer Westerwelle, Lafontaine und Trittin zu. Hier stimmte das Verhältnis und die Rollenverteilung zwischen Diskutanten (drei) und Moderatoren (zwei), so dass nicht zuletzt die Verdeutlichung der politischen Differenzen zwischen den drei Parteien weit besser gelang. Angesichts der deutlich geringeren Zuschauerzahl (4,2 Millionen) dürfte diese Sendung aber ebenfalls einen eher geringen Einfluss auf den Wahlausgang gehabt haben. Speziell die vielen politisch weniger interessierten, unentschiedenen Wähler dürften die Sendung kaum zur Entscheidungsfindung genutzt haben.

\section{Das Wahlergebnis im Detail}

\subsection{Wählerwanderung}

Wie tiefgreifend die Verwerfungen in der Wählerlandschaft waren, lässt sich an der Wählerwanderungsanalyse von Infratest dimap ermessen. Der weitaus größte Strom verlief von den Parteien zu den Nichtwählern. Von diesem Abstrom wurde keine Partei verschont, am meisten litt allerdings die SPD darunter, von deren Wählern per Saldo über zwei Millionen am Wahlsonntag zu Hause blieben. Aber auch Union (-1,08 Millionen) und Linke (-0,3 Millionen) gaben in erheblichem Umfang an das Nichtwählerlager ab, während FDP (-70.000) und Grüne (-30.000) noch relativ glimpflich davonkamen.

Bei dieser Bundestagswahl gab es aber auch mehr Wechsel zwischen den Parteien als bei den Wahlen zuvor. Fast jeder dritte Wähler (31 Prozent) gab an, diesmal mit der Zweitstimme eine andere Partei gewählt zu haben als noch vor vier Jahren. Die bei weitem größten Wählerbewegungen verliefen von den Unionsparteien in Richtung FDP sowie von der SPD zur Linken (per saldo jeweils 1,1 Millionen). Die SPD gab als einzige Partei an alle übrigen Wettbewerber Wähler ab. Rund zwei Millionen wechselten zur Linken und zu den Grünen, verblieben also im linken Lager. Rund 1,5 Millionen vollzogen einen Lagerwechsel und entschieden sich diesmal für Union oder FDP. Damit trugen sie maßgeblich zum Machtwechsel bei. Für die Union blieb der Zustrom von der SPD der einzige Zugewinn, ansonsten gaben CDU und CSU ebenfalls an alle anderen Parteien ab. Für den Wahlsieg von Schwarz-Gelb entscheidend war aber, dass die Verluste an den Wunschpartner FDP rund zehnmal so hoch ausfielen wie die an Grüne und Linke. Im generativen Wähleraustausch fiel die Bilanz der beiden Volksparteien aufgrund des jeweils hohen Anteils älterer Wähler

30 Ihnen wurde in der Berichterstattung durchweg ein übertriebener Hang zur Selbstdarstellung angekreidet. 
negativ aus. Von ihren ehemaligen Wählern verstarben mehr innerhalb der Wahlperiode, als sie an Zugewinnen von Erstwählern verzeichnen konnten. Positiv fiel dagegen die generative Bilanz der Grünen aus, deren Wähler immer noch überdurchschnittlich jung sind und die auch von dem nach wie vor großen Zulauf von Erstwählern profitierten. Dies galt im Übrigen ebenfalls für die Piratenpartei, die aber auch von allen anderen Parteien vor allem junge Wähler abzog.

Die Wanderungsbewegungen fielen in West und Ost recht unterschiedlich, teilweise sogar gegenläufig aus. So standen etwa bei der CDU zum Teil deutlichen Verlusten im Westen marginale Gewinne im Osten gegenüber, denn der starke Abstrom in Richtung FDP beschränkte sich weitestgehend auf die alten Bundesländer. Den Gegenpol bildet die Linkspartei, die ausschließlich im Westen real an Stimmen zulegte, im Osten dagegen stagnierte - was ihr wegen der geringen Wahlbeteiligung gleichwohl zu einem höheren Stimmenanteil verhalf.

\subsection{Wahlverhalten nach Regionen}

Noch deutlicher werden die mit der Bundestagswahl 2005 einhergehenden tiefgreifenden Veränderungen der politischen Landschaft, wenn man nicht nur das nationale Ergebnis, sondern auch die Resultate in den einzelnen Bundesländern oder Regionen betrachtet. Die Basistrends - große Verluste für die SPD, Gewinne für FDP, Grüne und Linke - verliefen zwar bundesweit weitgehend einheitlich; die Auswirkungen auf die regionalen Kräfteverhältnisse fielen aber recht unterschiedlich aus.

Die bundesweiten Verluste der CDU resultieren allein aus ihrem vergleichsweise schlechten Abschneiden im Westen, wo sie - mit Ausnahme von Bremen und dem Saarland - zum Teil erhebliche Verluste erlitt. Am deutlichsten fielen die Einbrüche in Schleswig-Holstein (-4,2 Prozentpunkte) und Baden-Württemberg $(-4,8)$ aus. In den neuen Bundesländern einschließlich Berlin konnten die Christdemokraten dagegen durchweg zulegen, am stärksten in Sachsen, Sachsen-Anhalt und Thüringen mit einem Plus von jeweils 5,5 Punkten.

Die CSU erlebte in Bayern mit einem Verlust von 6,7 Prozentpunkten einen neuerlichen Tiefschlag und fiel mit 42,5 Prozent noch hinter ihr schlechtes Ergebnis bei der Landtagswahl 2008 zurück. Damit liegt sie allerdings immer noch deutlich über dem höchsten Landesergebnis der Schwesterpartei, das die CDU diesmal mit 35,6 Prozent in Sachsen erzielte. Anders als bei früheren Wahlen ist die CSU aber nicht mehr „Wahlkreiskönig“: Der Wahlkreis mit dem höchsten Zweitstimmenanteil für eine Partei entfiel diesmal mit 54,5 Prozent auf die niedersächsische CDU im Kreis Cloppenburg-Vechta. Das schlechte Abschneiden der CSU im Freistaat korrespondiert mit den überdurchschnittlichen Verlusten der Union auf dem flachen Land (-4,2 Prozentpunkte) und in Regionen mit hohem Katholikenanteil $(-4,9)$. Da sie in ihren Hochburgen überdurchschnittlich verlor, sich aber in bislang schwachen Regionen weitgehend behaupten konnte, nivellierten sich die regionalen Unterschiede spürbar. Der Abstand zwischen dem Bundesland mit dem höchsten Ergebnisanteil für die Union (Bayern mit 42,5 Prozent) und dem Land mit dem schlechtesten Ergebnis (Berlin mit 22,8) reduzierte sich von 28,6 auf 19,7 Prozent, also um fast zehn Punkte. Auch auf Wahlkreisebene war die Berliner CDU mit Friedrichshain-Kreuzberg mit einem Stimmanteil von 11,9 Prozent Schlusslicht. 
Das gegenläufige Abschneiden der CDU im Osten und Westen Deutschlands trug ebenso zu einer leichten Annäherung des Wahlverhaltens in beiden Landesteilen bei wie das gute Abschneiden der Linkspartei in den alten Ländern. Die Unterschiede bleiben aber auch in der sechsten Wahlperiode nach der deutsch-deutschen Vereinigung weitgehend erhalten, die kumulierte Differenz der Parteianteile zwischen den alten und neuen Ländern beträgt immer noch 36,8 Punkte (2005: 43), wovon alleine 18,1 Punkte auf die Ost-West-Unterschiede bei der Linken entfallen.

\begin{tabular}{|l|rr|rr|c|}
\hline Tabelle 2: & \multicolumn{6}{c|}{$\begin{array}{c}\text { Bundestagswahl 2009 - Ergebnisse in West- und Ostdeutschland } \\
\text { (Zweitstimmenanteile in Prozent, in Klammern: Differenz 2009-2005) }\end{array}$} \\
\hline & \multicolumn{2}{|c|}{ West } & \multicolumn{2}{|c|}{ Ost } & Diff. West-Ost \\
\hline CDU/CSU & 34,9 & $(-2,8)$ & 29,5 & $(+3,9)$ & $+5,4$ \\
SPD & 24,2 & $(-11,0)$ & 18,3 & $(-12,5)$ & $+5,9$ \\
FDP & 15,4 & $(+5,2)$ & 11,0 & $(+2,7)$ & $+4,4$ \\
Die Linke & 8,3 & $(+3,5)$ & 26,4 & $(+3,1)$ & $-18,1$ \\
Grüne & 11,3 & $(+2,7)$ & 8,3 & $(+2,0)$ & $+3,0$ \\
\hline * Ost einschließlich Berlin. & \multicolumn{5}{|}{} \\
1 2005 = Linke.PDS. \\
Quelle: Bundeswahlleiter.
\end{tabular}

Die SPD verlor in allen 299 Wahlkreisen deutlich an Zweitstimmen. Die Einbußen in den einzelnen Ländern reichten von 8,6 Punkten im Saarland und in Bayern bis zu 15,2 Punkten in Mecklenburg-Vorpommern. Den höchsten Stimmenanteil erzielte sie diesmal mit 30,3 Prozent in dem kleinsten Bundesland Bremen. Der Stadtstaat war auch das einzige Land, wo sich die SPD bei dieser Wahl als stärkste Partei behaupten konnte - bei der Bundestagswahl 2005 war sie noch in zwölf Bundesländern stärkste Kraft. In Ostdeutschland fielen ihre Verluste noch etwas stärker aus als im Westen, so dass sie dort vom ersten auf den dritten Platz hinter CDU und Linkspartei zurückfiel. In allen fünf ostdeutschen Flächenländern erhielt die SPD diesmal weniger Stimmen als die Linke, in Sachsen kam ihr sogar die FDP mit einem Abstand von nur noch 1,3 Punkte gefährlich nahe. Dort verbuchten die Sozialdemokraten auch ihr schwächstes Wahlkreisergebnis (Erzgebirgskreis I mit 12,0 Prozent). Am besten schnitten sie noch im Wahlkreis Gelsenkirchen ab $(42,0)$. Anders als bei zurückliegenden Wahlen musste die SPD diesmal auch ohne jeglichen landsmannschaftlichen Rückenwind für ihren Spitzenkandidaten auskommen. In Niedersachsen, dem Herkunftsland Steinmeiers wie auch in Brandenburg, wo er als Spitzenkandidat auf der Landesliste antrat, verzeichnete sie zweistellige Verluste.

Die FDP legte in 298 Wahlkreisen zu und erreichte in 13 von 16 Bundesländern ein zweistelliges Wahlergebnis. Überdurchschnittliche Gewinne verzeichnete sie in den Ländern, in denen sie an der Regierung beteiligt ist. Den höchsten Zuwachs und das beste Ergebnis erzielte sie in ihrem Stammland Baden-Württemberg (18,8 Prozent), wo mit Rottweil-Tuttlingen auch ihre Wahlkreishochburg $(21,9)$ liegt. Dort wie in weiteren 14 Wahlkreisen (alle im Westen) wurde die FDP sogar zweitstärkste Partei vor der SPD. In acht Bundesländern ist sie drittstärkste Kraft, in Berlin rangiert sie dagegen hinter Grünen und Linken nur an fünfter Stelle. Die Streuung ihrer Höchst- und Niedrigstergebnisse ist sowohl in Bezug auf die Bundesländer (9,5 Punkte Abstand) wie die Wahlkreise $(15,8)$ die geringste unter den im 
Bundestag vertretenen Parteien - Beleg für die regionale Ausgeglichenheit der neuen FDP. Eine Sonderposition nimmt der Wahlkreis Dresden I ein, der einzige, in dem die FDP verlor. Dort fand 2005 wegen des Todes eines Direktkandidaten eine Nachwahl statt - ein Novum bei einer Bundestagswahl. Im Wissen um das Wahlergebnis im übrigen Bundesgebiet gaben viele eher der Union zugeneigte Wähler ihre Zweitstimme der FDP, um das schwarz-gelbe Lager insgesamt zu stärken ${ }^{31}$. Das Ergebnis war so einmalig wie das Zustandekommen: Mit einem Stimmenanteil von 16,6 Prozent fuhr die FDP - erstmals im Osten ihr bestes Wahlkreisergebnis ein. Die bei dieser Wahl erlittenen Verluste (-3,4 Punkte) sind also eher ein Schritt in Richtung Normalisierung, zumal das Ergebnis von Dresden I mit 13,2 Prozent noch über dem FDP-Schnitt in Ostdeutschland blieb.

Die Linkspartei verzeichnete eine ganze Reihe regional bemerkenswerter Ergebnisse. Auch sie legte in allen Wahlkreisen zu, mit einer einzigen Ausnahme (Wahlkreis Bautzen: -0,1 Punkte). In Sachsen-Anhalt übersprang sie mit 32,4 Prozent erstmals die 30-ProzentMarke und wurde dort - ebenfalls erstmalig in einem Bundesland - stärkste politische Kraft. Dies gelang ihr ebenfalls in Thüringen, wo sie mit einem Stimmenanteil von 28,5 Prozent anders als vier Wochen zuvor bei der Landtagswahl auch die CDU übertraf. In Berlin überwand sie in den Wahlkreisen Lichtenberg (41,2 Prozent) und Marzahn-Hellersdorf $(40,8)$ sogar die 40-Prozent-Marke und stellte damit auch auf Wahlkreisebene einen neuen Rekord auf. Die Linke legte aber auch im Westen deutlich um weitere 3,4 Punkte zu und schaffte mit einem bemerkenswerten Stimmenanteil von 8,3 Prozent schon im ersten Anlauf nach der Vereinigung von PDS und WASG den Sprung über die Fünf-ProzentHürde. Nur in sieben ausschließlich westdeutschen Wahlkreisen blieb sie unter fünf Prozent. Am schlechtesten schnitt sie mit 4,1 Prozent im einkommensstärksten oberbayerischen Wahlkreis Starnberg ab. Ihr bei weitem bestes Westergebnis erzielte sie mit 21,2 Prozent wie schon 2005 im Saarland, aber auch in Bremen (14,3 Prozent) und Hamburg $(11,2)$ kam sie auf zweistellige Ergebnisse. Insgesamt erhielt die Nachfolgepartei der PDS in den westlichen Bundesländern unter dem Strich sogar etwas mehr Stimmen als im Osten. Sie vollzog damit den bis vor fünf Jahren noch undenkbaren Schritt zu einer gesamtdeutschen Partei - ein Erfolg, der untrennbar mit dem Namen Oskar Lafontaine verbunden ist.

Wie FDP und Linke verzeichneten auch die Grünen in allen Bundesländern und in allen Wahlkreisen Zugewinne - mit ebenfalls nur einer einzigen Ausnahme, dem Wahlkreis Prignitz (-0,1 Punkte). Erstmals seit ihrem Bestehen übersprangen die Grünen auch in allen Bundesländern, im Westen sogar in allen Wahlkreisen die Fünf-Prozent-Hürde. Auf Landesebene erzielten sie ihre bei weitem besten Ergebnisse in den Stadtstaaten Berlin (17,4 Prozent), Hamburg $(15,6)$ und Bremen $(15,4)$, ihre schlechtesten in Sachsen-Anhalt $(5,1)$ und Mecklenburg-Vorpommern $(5,5)$. Am wenigsten konnten sie im Saarland hinzugewinnen $(+0,8$ Prozentpunkte) und in Hamburg $(+0,7)$, wo sie in einer schwarz-grünen Koalition mitregieren. Ihre Hochburgen liegen wie schon vor vier Jahren in Berlin (FriedrichshainKreuzberg: 27,4 Prozent), in Freiburg $(22,8)$ und in anderen westdeutschen Großstädten. Da die Grünen diesmal auch in den ostdeutschen Städten deutlich zulegten, nahm das Stadt/Land-Gefälle noch etwas zu. Den höchsten Zustrom an Wählern verzeichneten sie aber im ländlich geprägten Wahlkreis Lüchow-Dannenberg-Lüneburg (+6,1 Punkte), wo sich das von den Grünen bekämpfte Zwischenlager für atomare Abfälle befindet.

31 Vgl. Infratest dimap, Wahlreport Bundestagswahl 2005, Berlin, S. 13. 
In den Großstädten erwuchs ihnen in der Piratenpartei ein neuer Wettbewerber. Auch die Hochburg müssen sie sich mit ihnen teilen: Im alternativ geprägten Berliner Wahlkreis Friedrichshain-Kreuzberg überwanden die Piraten mit ihrem mit Abstand besten Ergebnis von 6,0 Prozent sogar die Fünf-Prozent-Hürde.

Die Machtverschiebung zu Gunsten der Union und zu Lasten der SPD findet auch in der Verteilung der Direktmandate ihren Niederschlag. Von den 299 Direktmandaten entfielen 218 auf die Unionsparteien, 68 mehr als bei der letzten Wahl. In Bayern, im Saarland und in Sachsen gewannen CSU und CDU alle Direktmandate. Das hervorragende Abschneiden auf dieser Ebene gepaart mit dem relativ bescheidenen Gesamtergebnis führte aufgrund des noch geltenden Wahlrechts zu der bislang noch nie erreichten Zahl von 24 Überhangmandaten (+16). Insgesamt besteht die Unionsfraktion nun zu 91 Prozent aus siegreichen Direktkandidaten (2005 waren es nur 66 Prozent) - keine leichte Aufgabe für die Fraktionsführung von CDU/CSU. Ganz anders verlief die Entwicklung bei der SPD, die nur noch 64 Mandate direkt gewinnen konnte - ein Minus von 86. In der SPD-Fraktion verringerte sich damit der Anteil an Wahlkreissiegern von 68 auf 44 Prozent. In den neuen Bundesländern erzielte die SPD nur noch in Brandenburg fünf Direktmandate, dafür legte die Linke im Osten mit insgesamt sechzehn Direktmandaten (+13) deutlich zu. Sie errang in allen östlichen Bundesländern außer Sachsen mindestens ein Direktmandat. Ein Wahlkreis fiel wiederum an die Grünen: In Friedrichshain-Kreuzberg wurde zum dritten Mal hintereinander Christian Ströbele direkt und mit höherem Stimmanteil $(+3,4$ Punkte) gewählt. In Stuttgart I, wo sich die Grünen ebenfalls Hoffnungen auf ein Direktmandat machten, unterlag ihr Parteichef Cem Özdemir letztlich doch mit deutlichem Abstand von 4,5 Punkten dem CDU-Kandidaten. Immerhin erzielte Özdemir in seinem Wahlkreis 7,9 Prozentpunkte mehr Erststimmen als seine Partei Zweitstimmen. Damit rangierte er bei den Grünen als Wahlkreismagnet direkt hinter Ströbele, der mit einem Plus von 19,3 Punkten den größten Erststimmenüberhang aller 299 Direktkandidaten einfuhr. Auf den nächsten vier Plätzen folgten drei Unionspolitiker: Karl-Theodor zu Guttenberg (+18,6 Punkte), Wolfgang Bosbach $(+14,5)$ und Julia Klöckner, die designierte Herausfordererin der CDU für Kurt Beck in Rheinland-Pfalz $(+13,9)$. Dazwischen schob sich der „Erststimmenkönig“ der SPD Axel Berg mit einem Plus von 15,8 Punkten. Er verpasste damit aber den Einzug in den Bundestag, da er in München Nord dem CSU-Bewerber um ganze 1.123 Stimmen unterlag und auf der SPD-Landesliste nicht ausreichend abgesichert war. Erfolgreicher war Gregor Gysi, der sein Direktmandat für die Linke mit großem Vorsprung vor dem CDU-Kandidaten und mit dem größten Erststimmenüberhang der Linken $(+11,0$ Punkte) gewann. Bei der FDP war der bayerische Abgeordnete Max Stadler Wahlkreisbester $(+3,4)$. Sein Erststimmenanteil von 18,9 Prozent wurde nur übertroffen von seinem Parteivorsitzenden Westerwelle, der im Wahlkreis Bonn 19,1 Prozent der Erststimmen erhielt. Von den Spitzenkandidaten erzielte Angela Merkel den höchsten Erststimmenüberhang (+12,1 Punkte), gefolgt von Peter Ramsauer $(+5,8)$ und Frank-Walter Steinmeier (+5,2).

\subsection{Wahlverhalten nach Bevölkerungsgruppen}

Auch bei den verschiedenen Bevölkerungsgruppen herrschen weitgehend einheitliche Trends im Wahlverhalten vor: Die SPD verlor ausnahmslos in allen Gruppen, während FDP, Linke und Grüne überall zumindest anteilsmäßig zulegen konnten. Einzig bei der 
Union fällt die Bilanz wiederum gemischt aus: Gewinne in der einen Bevölkerungsgruppe stehen zum Teil deutlichen Verlusten in anderen gegenüber. Gewonnen hat die Union in zwei Gruppen, bei denen sie sich in den zurückliegenden Wahlen sehr schwer getan hat: bei Ostdeutschen und bei jungen Frauen. Hier zog sie erfolgreich von der SPD Wähler ab. Dem stehen Verluste bei Männern in allen Altersgruppen gegenüber, die höchsten bei den 45- bis 59-jährigen (-6 Punkte). Diese Verluste wurden aber durch die überdurchschnittlichen Gewinne der FDP bei der männlichen Wählerschaft mehr als ausgeglichen.

Aufgrund der massiven kohortenübergreifenden Verluste der SPD ging die Union in allen Altersgruppen und bei Frauen wie Männern als klar stärkste Kraft hervor. Auffallend ist dabei das gegenläufige Abschneiden der CDU in Ost und West bei Frauen und Männern: Deutlichen Gewinnen bei den Frauen in den neuen Ländern (+7 Punkte, bei den 25- bis 34-jährigen Frauen sogar +10) stehen fast ebenso deutliche Verluste bei den Männern in den alten Bundesländern (-5) gegenüber. Bei den männlichen Wählern im Osten und den weiblichen im Westen blieben die Anteilswerte stabil. Die Altersstruktur der Unionswählerschaft blieb dabei weitgehend erhalten: Ihren bei weitem höchsten Stimmenanteil erreichte sie bei den über 60-Jährigen mit 43 Prozent (bei den über 70-Jährigen sogar 47 Prozent), ihren niedrigsten bei den 18- bis 24-Jährigen mit nur 25 Prozent, womit sie aber immer noch klar vor der SPD (18 Prozent) lag. Die Sozialdemokraten müssen vor allem in den unteren und mittleren Altersgruppen und bei den Höhergebildeten um ihren zweiten Platz in der Parteienhierarchie fürchten. Bei den Wählerinnen zwischen 18 und 44 Jahren lagen die Grünen praktisch gleichauf, bei Frauen mit Abitur in dieser Altersgruppe sogar deutlich vor der SPD. Bei den männlichen Wählern unter 45 Jahren konkurriert die SPD mit der FDP um Platz 2, wobei sie wiederum bei den Abiturienten erstmals deutlich hinter den Liberalen rangierte.

Die neue Offenheit im Wahlverhalten lässt sich am besten an den Ergebnissen der Jungwähler beobachten. Bei den 18- bis 24-Jährigen erlitt die SPD ihre bei weitem höchsten Verluste, ihr Anteil in dieser Altersgruppe halbierte sich von 38 auf 18 Prozent. Von der Schwäche der SPD profitierte die Union nur bei den jungen Frauen, bei den 18- 24-Jährigen Männern erlitt sie dagegen deutliche Verluste. Mit 23 Prozent bei den männlichen und 28 Prozent bei den weiblichen Jungwählern ist aber auch die Union alles andere als die dominierende Kraft. Die SPD ist mit 18 Prozent nur noch eine Partei mittlerer Stärke, zusammen mit der FDP, den Grünen (jeweils 15 Prozent) und der Linken (11 Prozent). Sichtbarstes Zeichen für die neue Offenheit ist das Abschneiden der Piratenpartei. Sie erreichte bei den Jungwählern auf Anhieb und mit nur geringem Vorlauf einen Stimmenanteil von 9 Prozent, bei den männlichen Jungwählern lag sie mit 12 Prozent sogar Kopf an Kopf mit Grünen und Linken. Zwischen der Union als stärkster und den Piraten als sechststärkster Partei in dieser Alterskohorte liegen gerade einmal 16 Punkte. Die Piratenpartei ist im Übrigen ein gesamtdeutsches Phänomen, sie erzielte in Ost- und Westdeutschland fast identische Ergebnisse. Die Schattenseite dieser neuen politischen Offenheit der jungen Generation - vor allem der jungen Männer - ist eine deutlich größere Affinität auch zu rechtsradikalen Parteien. Diese beschränkt sich nach wie vor weitgehend auf die neuen Bundesländer, wo die NPD mit 9,3 Prozent fast wieder ein zweistelliges Ergebnis einfuhr (2005 waren es noch knapp 13 Prozent). 


\begin{tabular}{|c|c|c|c|c|c|c|c|c|c|c|c|}
\hline \multicolumn{12}{|c|}{$\begin{aligned} \text { Tabelle 3: Bundestagswabl 2009, Wablverhalten nach Alter und Geschlecht } \\
\text { (Stimmenanteile in Prozent; Veränderungen zu } 2005 \text { in Prozentpunkten) }\end{aligned}$} \\
\hline & \multicolumn{2}{|c|}{ CDU/CSU } & \multicolumn{2}{|c|}{ SPD } & \multicolumn{2}{|c|}{ FDP } & \multicolumn{2}{|c|}{ Die Linke* } & \multicolumn{2}{|c|}{ Grüne } & \multirow{2}{*}{\begin{tabular}{|c|} 
Pirater \\
$\%$ \\
\end{tabular}} \\
\hline & $\%$ & Diff. & $\%$ & Diff. & $\%$ & Diff. & $\%$ & Diff. & $\%$ & Diff. & \\
\hline Ergebnis & 33,8 & $-1,4$ & 23,0 & $-11,2$ & 14,6 & $+4,7$ & 11,9 & $+3,2$ & 10,7 & $+2,6$ & 2,0 \\
\hline 18-24 Jahre & 25 & -1 & 18 & -20 & 15 & +4 & 11 & +3 & 15 & +5 & 9 \\
\hline $25-34$ & 29 & -0 & 17 & -16 & 18 & +5 & 12 & +3 & 14 & +3 & 4 \\
\hline $35-44$ & 30 & -1 & 21 & -13 & 16 & +6 & 12 & +3 & 15 & +3 & 2 \\
\hline $45-59$ & 31 & -3 & 24 & -10 & 14 & +5 & 14 & +4 & 12 & +3 & 1 \\
\hline 60 und älter & 43 & -0 & 27 & -7 & 12 & +3 & 10 & +3 & 5 & +1 & 0 \\
\hline Frauen & 36 & +1 & 23 & -13 & 13 & +4 & 11 & +3 & 12 & +3 & 1 \\
\hline 18-24 Jahre & 28 & +2 & 18 & -21 & 12 & +3 & 12 & +4 & 19 & +8 & 5 \\
\hline $25-34$ & 32 & +3 & 18 & -17 & 16 & +5 & 12 & +3 & 16 & +5 & 2 \\
\hline $35-44$ & 32 & +1 & 21 & -14 & 14 & +5 & 11 & +3 & 17 & +4 & 1 \\
\hline $45-59$ & 32 & -1 & 24 & -12 & 13 & +5 & 13 & +4 & 13 & +3 & 1 \\
\hline 60 und älter & 45 & +3 & 27 & -9 & 11 & +3 & 9 & +3 & 6 & +1 & 0 \\
\hline Männer & 32 & -4 & 23 & -10 & 16 & +6 & 13 & +3 & 9 & +2 & 3 \\
\hline 18-24 Jahre & 23 & -3 & 17 & -19 & 17 & +6 & 11 & +3 & 12 & +3 & 12 \\
\hline $25-34$ & 27 & -3 & 17 & -14 & 20 & +5 & 12 & +3 & 11 & +2 & 6 \\
\hline $35-44$ & 29 & -4 & 21 & -12 & 18 & +7 & 12 & +3 & 12 & +2 & 3 \\
\hline $45-59$ & 29 & -6 & 25 & -9 & 15 & +6 & 16 & +4 & 11 & +3 & 1 \\
\hline 60 und älter & 41 & -3 & 28 & -5 & 14 & +4 & 12 & +3 & 4 & +1 & 0 \\
\hline
\end{tabular}

Ihren Status als dominierende Volksparteien können Union und SPD nur noch bei den über 70-Jährigen und bei den über 60-Jährigen behaupten mit Stimmanteilen von 75 beziehungsweise 70 Prozent; bei den Jungwählern liegen sie zusammen erstmals und klar unter der Fünfzigprozentmarke. Dieser Bedeutungsverlust der Volksparteien macht auch vor den klassischen Unterstützermilieus in Union und SPD nicht Halt. CDU und CSU verloren in ihrer Kerngruppe, dem katholischen Milieu, leicht überdurchschnittlich (-3 Punkte), konnten sich aber mit einem Anteil von 45 Prozent wenigstens noch als eindeutig stärkste Kraft behaupten. Drastischer verläuft die Auflösung traditioneller Parteibindungen bei den Gewerkschaftsmitgliedern, der Kernwählerschaft der SPD. Von ihnen entschieden sich nur noch 34 Prozent für die SPD, 13 Prozentpunkte weniger als 2005. Als Auffangbecken für die von der SPD abgewanderten Wähler fungierten nicht nur die beiden Wettbewerber im linken Lager - die Linke mit 15 Prozent (+3 Punkte) und die Grünen mit 11 Prozent $(+3)$-, sondern auch die Parteien rechts von der Mitte. Die Union kam bei den Gewerkschaftern auf 24 Prozent (+1 Punkt) und die FDP auf 9 Prozent (+4) - Parteien, die früher in diesem Milieu eher als politischer Gegner wahrgenommen wurden. Das Wahlverhalten hat sich damit weiter von dem alten Klassenschema gelöst, wie man an dem ausge- 
prägt volatilen Abstimmungsergebnis in der Arbeiterschaft und bei den Selbständigen erkennen kann. In beiden Gruppen erlitten die Volksparteien zum Teil herbe Verluste, während FDP, Linke und Grüne kräftig zulegten. Bei den Selbständigen konnte sich die Union mit 33 Prozent nur knapp vor der FDP (26 Prozent) als stärkste Kraft behaupten, während die SPD mit 14 Prozent gleichauf mit den Grünen auf den dritten Platz zurückfiel. Die Arbeiter votierten 2009 sogar fast gleichermaßen für linke Parteien (49 Prozent für SPD, Linke und Grüne) wie für rechte (41 Prozent für CDU und FDP). Einzig die Arbeitslosen wählten mehrheitlich links, mit 25 Prozent war in dieser Gruppe sogar erstmals die Linke stärkste Partei. Dies gelang den Linken ansonsten nur noch bei den konfessionell ungebundenen Wählern, wo die SPD massive Verluste erlitt und noch hinter die Union zurückfiel.

\subsection{Wahlmotive}

Im Rahmen der ARD-Wahltagsbefragung von Infratest dimap wird seit der Bundestagswahl 2002 erhoben, ob die Wahlentscheidung eher themenorientiert oder personenbezogen erfolgt ist oder ob die Parteibindung ausschlaggebend war. Die Befunde zeigen über die Zeit, dass von diesen drei Faktoren die Parteibindung den geringsten Einfluss hat. Bei der Bundestagswahl 2009 ließen sich nur 18 Prozent der Wähler davon leiten, 2005 waren es 20 Prozent $^{32}$. Die inhaltlichen Positionen werden über alle Parteien hinweg am häufigsten als wichtiger Entscheidungsgrund angegeben (55 Prozent), gefolgt vom personellen Angebot der Parteien (22 Prozent). Generell spielen Parteibindung und Spitzenkandidaten für die Volksparteien eine deutlich größere Rolle als für die kleinen Parteien, für deren Wähler in erster Linie inhaltliche Aspekte im Vordergrund stehen. Angesichts einer zunehmenden Unübersichtlichkeit des Parteienmarktes kommt darüber hinaus koalitionspolitischen strategisch-taktischen Überlegungen wachsende Bedeutung zu. Das Gewicht der einzelnen Faktoren fällt dabei von Partei zu Partei sehr unterschiedlich aus.

\section{Unionswähler}

Für 40 Prozent der Unionsanhänger waren nach eigenem Bekunden sachpolitische Erwägungen ausschlaggebend für ihre Wahlentscheidung. In erster Linie standen dabei wirtschaftspolitische Aspekte im Vordergrund, gefolgt von der Arbeitsmarktpolitik, der Ausrichtung der Politik auf soziale Gerechtigkeit und der Steuerpolitik. In den Feldern Wirtschaft und Steuern wird die Union auch als die mit Abstand kompetenteste Partei angesehen, wobei ihr in den letzten Jahren in der FDP eine ernsthafte Konkurrentin erwachsen ist. CDU und CSU wird darüber hinaus die größte Lösungskompetenz bei der inneren Sicherheit sowie - neuerdings - in der Familienpolitik zugeschrieben. Aufgrund der unter von der Leyen betriebenen völligen Neuausrichtung gelang es der CDU, in diesem sozial-

32 Die klassische Frage zur Parteibindung kommt zu anderen Zahlen, aber vergleichbaren Befunden. Unmittelbar vor der Bundestagswahl gaben im DeutschlandTrend 54 Prozent der Wahlberechtigten an, einer Partei zuzuneigen - 22 Prozent stark, 29 Prozent mäßig und 3 Prozent schwach -, Tendenz ebenfalls sinkend, 2000 bezeichneten sich noch 62 Prozent als parteilich gebunden. 
und kulturpolitisch relevanten Politikfeld erstmals seit langem wieder mehr Vertrauen zu binden als die SPD. Mit einer modernen Familienpolitik wurde die Partei für viele junge, berufstätige Frauen wieder wählbar. Die am traditionellen Familienbild festhaltende CSU hat hingegen in dieser Gruppe nach wie vor Akzeptanzprobleme und verlor bei den 18- bis 34-jährigen Frauen acht Prozentpunkte, während die CDU in dieser Gruppe gegen den Trend zulegen konnte. Am Ende trug auch die Wirtschafts- und Finanzkrise entscheidend dazu bei, dass die Union wieder deutlich stärkste Partei wurde, denn ihr wurde eher als allen anderen Parteien zugetraut, die Krise zu bewältigen und die Zukunft erfolgreich zu gestalten.

Die Union wies aber nicht nur in sachpolitischer Hinsicht klare Vorteile gegenüber ihrem Koalitionspartner und den Oppositionsparteien auf, auch in personeller Hinsicht wusste sie weit mehr zu überzeugen. In der Auseinandersetzung mit der SPD machte der Kanzlerbonus, den diesmal die Union innehatte, den entscheidenden Unterschied zu 2002 und 2005 aus. Damals verstand es die SPD, mit der Beliebtheit Gerhard Schröders die wachsende Unzufriedenheit mit Rot-Grün und den Kompetenzvorsprung von CDU/CSU in vielen Bereichen auszugleichen. Mit einer CDU-Kanzlerin an der Spitze war die Union immer präsenter und vermochte so, die Erfolge der Regierung gerade bei der Bekämpfung der Wirtschaftskrise weitgehend auf ihrem Konto zu verbuchen. Vor allem in den diversen internationalen Gipfeltreffen verstand sie es, sich als weltweit angesehene Verhandlungspartnerin zu profilieren. Als Außenminister erreichte zwar der SPD-Spitzenkandidat ähnliche Zustimmungswerte wie Merkel, als Kanzler zog eine deutliche Mehrheit der Deutschen aber Merkel vor. Am Ende sprachen sich 51 Prozent der Wahlberechtigten für Merkel und 36 Prozent für Steinmeier aus ${ }^{33}$. Die CDU-Chefin lag im direkten Vergleich mit ihrem Herausforderer auch in punkto Führungskraft, Kompetenz und Glaubwürdigkeit bis zum Schluss klar vorn. Entscheidender für den Wahlausgang dürfte allerdings die ihr zugeschriebene soziale Kompetenz gewesen $\operatorname{sein}^{34}$. Mit ihrer zwar unausgesprochenen, aber in ihrem Handeln deutlich werdenden Abkehr von den Leipziger Beschlüssen gelang es Merkel, dem Verdacht entgegenzuwirken, der Union seien die sozialen Belange der Bürger weitgehend egal. Sie stand zweifelnden SPD-Wählern gleichsam persönlich als Gewähr dafür, dass sich auch eine schwarz-gelbe Regierung dem Ziel sozialer Gerechtigkeit verpflichtet fühlen würde. Ihr hohes persönliches Ansehen dokumentiert sich nicht zuletzt darin, dass 32 Prozent der Unionswähler insgesamt und sogar 38 Prozent der zugewanderten Wähler angaben, sich hauptsächlich wegen Merkel für die Union entschieden zu haben ${ }^{35}$.

Koalitionspolitisch hatten die Unionswähler eine klare Perspektive, denn die Unionsparteien ließen - ungeachtet einiger Scharmützel vor allem zwischen CSU und FDP - keinerlei Zweifel erkennen, mit den Liberalen zusammengehen zu wollen. Dies entsprach auch der Koalitionspräferenz ihrer Anhänger, denn in der ARD-Wahltagsbefragung sprachen sich sieben von zehn Unionswählern für ein Bündnis mit den Liberalen aus.

33 Vgl. Infratest dimap, a.a.O. (Fn. 19), S. 47.

34 In einer im März 2009 für den Bericht aus Berlin durchgeführten Umfrage von Infratest dimap lag sie auch in dieser Hinsicht vor Steinmeier.

35 ARD / Infratest dimap, Wahltagsbefragung Bundestagswahl 2009. 


\section{FDP-Wähler}

Ihr bislang bestes Abschneiden bei einer Bundestagswahl verdankte die FDP vor allem ihrer konsequent verfolgten Profilierung als Hüterin marktwirtschaftlicher Prinzipien und Steuersenkungspartei. Jeder fünfte Bundesbürger wies ihr in der Steuerpolitik die größte Kompetenz zu (2005 waren es gerade einmal sechs Prozent), und jeder zweite stimmte der Aussage zu, die FDP setze sich am konsequentesten für notwendige Reformen im Steuersystem ein. Die Finanzierbarkeit der versprochenen Senkungen wurde zwar von einer Mehrheit der Bürger in Frage gestellt, zahlreiche Selbständige, aber auch eine wachsende Zahl von jungen Angestellten bis hin zu gut verdienenden Arbeitern, die alle ihre Steuerbelastung als zu hoch und auch als ungerecht ansahen, identifizierten sich jedoch mit dieser Forderung. Der Großteil der von anderen Parteien, vor allem von der Union zur FDP abgewanderten Wähler gab in der ARD-Wahltagsbefragung für diesen Wechsel wirtschafts- und steuerpolitische Gründe an. Gegenüber 2005 gewann die FDP aber auch in der Wirtschafts-, der Bildungs-, der Gesundheits- sowie der Arbeitsmarktpolitik deutlich an Profil und erzielte dort fast überall zweistellige Kompetenzwerte. Und immerhin acht Prozent sahen sie als die Partei an, bei der auch das Thema soziale Gerechtigkeit am besten aufgehoben sei.

An Profil gewann auch ihr Parteivorsitzender Westerwelle, auch wenn er nach wie vor stark polarisiert. Mit seiner Arbeit als Fraktionschef der FDP zeigten sich 47 Prozent zufrieden, 50 Prozent dagegen unzufrieden. Bei den eigenen Anhängern erzielte er mit 93 Prozent einen vergleichbaren Zustimmungswert wie Merkel bei den Unionsanhängern. Einer Mehrheit der Deutschen gilt er heute als ein ernsthafter, verlässlicher und glaubwürdiger Politiker, einzig seine Eignung als Außenminister wurde vor der Wahl in Zweifel gezogen ${ }^{36}$. Da die Liberalen von Union und SPD als Koalitionspartner umworben wurden, kam ihnen bei dieser Wahl eine Schlüsselrolle zu. Deshalb verwundert es nicht, dass sich kurz vor der Wahl 57 Prozent der Bürger für eine Beteiligung der Liberalen an der Bundesregierung aussprachen, darunter nicht nur fast alle Anhänger von FDP und Union, sondern auch ein Drittel der Anhänger der SPD und jeder fünfte der Grünen, die die Liberalen für eine Regierungsmehrheit gebraucht hätten. Die Präferenz der FDP-Wähler galt allerdings eindeutig einer schwarz-gelben Koalition, für die sich 87 Prozent aussprachen. Nur ganze acht Prozent wollten mit ihrem Votum für die FDP einer Ampelkoalition zur Mehrheit verhelfen ${ }^{37}$. Diese enge Anlehnung an die Union fand ihren stärksten Ausdruck im lagerkonformen Stimmensplitting: Jeder zweite FDP-Zweitstimmenwähler gab seine Erststimme der CDU oder $\mathrm{CSU}^{38}$, was mit zu der hohen Zahl an Überhangmandaten für die Union beitrug. Wie schon $2005^{39}$ waren für viele FDP-Wähler koalitionspolitische Erwägungen ein entscheidendes Motiv, den Freidemokraten und nicht der CDU oder CSU die Zweitstimme zu geben.

36 Vgl. Infratest dimap im Auftrag der Fernsehsendung „Beckmann“ vom Januar und November 2009, http://www.infratest-dimap.de/umfragen-analysen/bundesweit/umfragen/aktuell/geringerzuspruch-fuer-westerwelle-als-aussenminister (Abruf am 1. Februar 2010) und http://www.infratest-dimap.de/de/umfragen-analysen/bundesweit/umfragen/aktuell/ein-drittel-begruesst-besetzung-des-auswaertigen-amts-mit-westerwelle-mehrheit-haelt-deutsche-afgha (Abruf am 1. Februar 2010).

37 Vgl. Infratest dimap, a.a.O. (Fn. 19), S. 64.

38 Ebenda, S. 65.

39 Vgl. hierzu Bernhard Weßels, Geheime Wahl - was Meinungsforscher vor dem 18. September nicht wissen konnten, in: WZB-Mitteilungen, H. 110 (Dezember 2005), S. 7 - 10. 
SPD-Wähler

In Anbetracht der massiven Stimmenverluste der SPD bedarf weniger die Frage, warum sie gewählt wurde, einer Antwort, sondern warum sie von so vielen ehemaligen Wählern nicht mehr gewählt wurde. Ihr Absturz kam nicht überraschend, denn der Wählerrückhalt brach nicht erst in dieser Wahlperiode ein, wie der Kurvenverlauf für die SPD in der Sonntagsfrage verdeutlicht, er vollzog sich in Raten, beginnend schon im Jahre 1999.

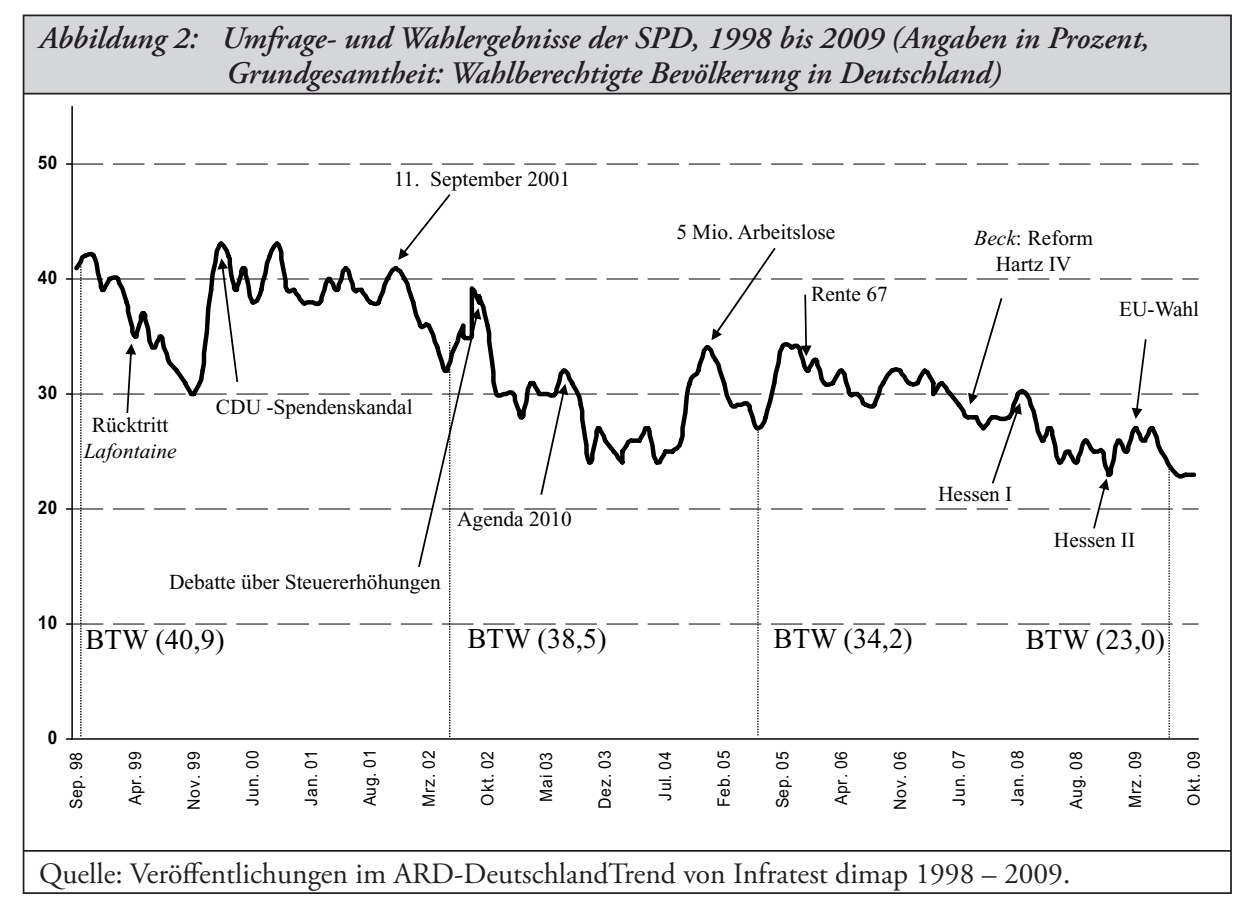

Die Bruchstellen im Wählervertrauen in die SPD verlaufen zum einen entlang der Turbulenzen an der Führungsspitze beziehungsweise der Versuche zur Bildung einer rot-rot-grünen Regierung in Hessen, zum anderen sind sie bedingt durch sachpolitische Entscheidungen (Steuerdiskussion/„Wählertäuschung“, Agenda 2010/Hartz IV, Rente mit 67). Letztere führten zu einer sukzessiven Erosion ihres Markenkerns „soziale Gerechtigkeit“ ${ }^{40}$, die für die SPD-Wähler auch bei dieser Wahl das wichtigste Motiv für ihre Wahlentscheidung war. In der Großen Koalition konnten zwar die Sozialdemokraten ihre Kompetenzführerschaft in diesem Politikfeld einigermaßen behaupten, ohne aber an die hohen Werte früherer Jahre anknüpfen zu können. Immer mehr Bürger weisen anderen Parteien in dieser Hinsicht größere Fähigkeiten zu, insbesondere in der Linken ist ihr in diesem Bereich ein ernsthafter Wettbewerber erwachsen. Speziell ältere Arbeitnehmer und darunter vor allem Arbeiter sahen in der Politik der SPD zunehmend eine Gefährdung ihrer sozialen Sicherung. Der Vorwurf, die Partei habe ihre Grundprinzipien in Sachen sozialer Gerechtigkeit aufgegeben,

40 Dies bestätigt der weitgehend parallele Kurvenverlauf der Parteisympathie mit der Kompetenzzuschreibung der SPD im Hinblick auf soziale Gerechtigkeit im ARD-DeutschlandTrend. 
war am Ende der dritten Regierungsperiode erstaunlicherweise noch weiter verbreitet als vier Jahre zuvor (67 Prozent Zustimmung gegenüber 55 Prozent im Jahre 2005) und stellte eine der wesentlichen Ursachen für die massiven Wählerverluste der SPD ${ }^{41}$ dar.

In ihrer elfährigen Regierungszeit hatte die SPD aber nicht nur auf der sozialen Dimension deutlich an Kompetenzzuschreibung eingebüßt, sondern auch auf den ökonomischen und den kulturellen Politikfeldern ${ }^{42}$. In Zeiten der globalen Krise vertraute nur noch ein Fünftel der Wählerschaft in ihre wirtschaftspolitischen Konzepte - in den beiden rot-grünen Regierungsperioden waren es immerhin noch gut ein Drittel. Selbst mehr als die Hälfte ihrer eigenen Wähler plagten Zweifel, ob die Sozialdemokraten eher als die Union in der Lage seien, die Wirtschafts- und Finanzkrise zu bewältigen. Viele zogen daraus Konsequenzen und machten diesmal ihr Kreuz bei der CDU/CSU oder der FDP: Zwei Drittel der insgesamt 1,5 Millionen SPD-Abwanderer in Richtung Union oder FDP begründeten in der Wahltagsbefragung diesen Schritt mit wirtschafts- oder steuerpolitischen Erwägungen. Unter dem Strich wanderten zwar weit mehr Wähler der SPD wegen empfundener Gerechtigkeitsdefizite in Richtung Linkspartei und Grüne ab oder blieben der Wahl fern; der zahlenmäßig zwar kleinere, von ökonomischen Motiven geprägte Abstrom zu Union und FDP war aber für den Wahlausgang bedeutsamer, weil er lagerübergreifend verlief. Befördert wurde dieser Strom zudem dadurch, dass die SPD auch in der Familienpolitik und auf dem zunehmend wichtigen Feld der Integrationspolitik hinter die Union zurückfiel. In der Bildungspolitik konnte sie sich zwar behaupten und bekam dafür ebensoviel Zustimmung wie die Unionsparteien, aber nachdem die Große Koalition die Zuständigkeit dafür weitestgehend an die Länder abgegeben hatte, spielte dieses Thema im Wahlkampf kaum eine Rolle. Die Bürger maßen der Bildungspolitik jedoch bei dieser Bundestagswahl sogar weit mehr Bedeutung bei als noch $2005^{43}$. Mit der Aussparung dieses Themas und der Vernachlässigung der Familien- und Integrationspolitik vergab die SPD eine Chance, vor allem junge und weibliche Wähler zu erreichen und Unentschiedene zu mobilisieren.

Eine Volkspartei wie die SPD hat sehr heterogene Wählergruppen mit zum Teil widersprüchlichen Interessen abzudecken. Dabei kommt ihren Spitzenpolitikern eine entscheidende Integrationsfunktion zu. Dafür ist nicht nur die Akzeptanz der jeweiligen Personen wichtig, sondern auch ein Mindestmaß an Beständigkeit an der Spitze. In dieser Hinsicht hat die SPD ihrer Wählerklientel seit ihrem Regierungsantritt viel zugemutet. In den elf Jahren ihrer Regierungsbeteiligung wechselte sie fünfmal den Vorsitzenden. Diese Wechsel an der Spitze waren häufig begleitet von Flügelkämpfen, was nach außen nachhaltig den Eindruck mangelnder Geschlossenheit vermittelte, und die Bindung ihrer Wähler an die Partei zusätzlich untergrub. Insofern hatte Steinmeier als SPD-Spitzenkandidat bei der Bundestagswahl 2009 eine extrem schwierige Ausgangsposition. Aufgrund des fehlenden Kanzlerbonus vermochte er auch nicht in dem Umfang wie Schröder in den Wahlen zuvor, die Rolle der Wahllokomotive für die SPD zu spielen. Diesmal gaben nur 22 Prozent der SPD wegen des Spitzenkandidaten ihre Stimme, immerhin jeder fünfte SPD-Wähler zeigte noch

41 Vgl. Infratest dimap, a.a.O. (Fn. 19), S. 41.

42 Zur Bedeutung der drei Dimensionen vgl. Joachim Raschke / Ralf Tils, Politische Strategie. Eine Grundlegung, Wiesbaden 2007.

43 In der ARD-Wahltagsbefragung nannten 22 Prozent der Wähler, 9 Punkte mehr als 2005, die Bildungspolitik als für ihre Wahlentscheidung entscheidend. Vgl. Infratest dimap, a.a.O. (Fn. 19), S. 64. 


\begin{tabular}{|c|c|c|c|c|c|}
\hline \multicolumn{6}{|c|}{ Abbildung 3: Wahlentscheidende Aspekte (Angaben in Prozent, Mehrfachnennungen möglich) } \\
\hline & \multicolumn{5}{|c|}{ Verluste von SPD an ... } \\
\hline & Alle & Union & FDP & Die Linke & Grüne* \\
\hline Wirtschaftspolitik & 39 & 46 & 48 & 25 & 23 \\
\hline Arbeitsmarktpolitik & 27 & 31 & 34 & 32 & 22 \\
\hline Steuerpolitik & 15 & 14 & 31 & 13 & 40 \\
\hline Soziale Gerechtigkeit & 34 & 24 & 25 & 63 & 33 \\
\hline
\end{tabular}

* Wichtigster wahlentscheidender Aspekt für Verluste der SPD an die Grünen waren Umwelt- und Klimapolitik mit 55 Prozent.

Quelle: ARD / Infratest dimap, Wahltagsbefragung Bundestagswahl 2009.

in der Schlusswoche eine Präferenz für Merkel als Kanzlerin. Steinmeier gelang es zudem nur unzureichend, die große Zahl unentschiedener SPD-Wähler zu erreichen, die letztlich in großer Zahl der Wahl fern blieben.

Die frühe und alternativlose Festlegung der SPD auf eine Ampelkoalition war wegen der vehementen Ablehnung durch die FDP nicht nur wenig glaubwürdig, sondern sie entsprach auch nicht dem mehrheitlichen Willen der eigenen Wähler. In der Wahltagsbefragung sprachen sich nur 32 Prozent der SPD-Wähler dafür aus, exakt ein Drittel votierte für die Fortsetzung die Großen Koalition, die bei den SPD-Anhängern von allen Optionen die größte Akzeptanz fand ${ }^{44}$. Jeder Vierte optierte für eine rot-rot-grüne Koalition, die aber von der SPD-Spitze rundweg abgelehnt worden war. Der letztlich gescheiterte Versuch, in Hessen ein solches Bündnis zu etablieren und die Diskussionen nach den Landtagswahlen im Saarland und in Thüringen über ein eventuelles Zusammengehen mit der Linken führten aber dazu, dass dieser Beschluss immer wieder vom politischen Gegner in Zweifel gezogen werden konnte. Da eine rot-rot-grüne Option auch in der SPD-Klientel deutlich mehr Ablehnung als Zuspruch erfuhr, trug dies zu einer nicht zu unterschätzenden Verunsicherung bei, denn immerhin jeder vierte SPD-Anhänger misstraute bis zum Schluss der Absage der Sozialdemokraten an ein Zusammengehen mit der Linken.

\section{Grünen-Wähler}

Die Grünen traten mit einem klar strukturierten politischen Programm an, dessen Schwerpunkt auf Ökologie und nachhaltigem Wachstum lag. Damit trafen sie die Stimmung ihrer Klientel, für die die Umweltpolitik in Zeiten des Klimawandels noch an Bedeutung gewonnen hat: Für 66 Prozent der Grünen-Wähler standen bei der Wahlentscheidung umweltpo-

44 Vgl. ARD / Infratest dimap, a.a.O. (Fn. 35); Infratest dimap, a.a.O. (Fn. 19), S. 64. Die Große Koalition hätten 57 Prozent der SPD-Anhänger gut gefunden, eine Ampelkoalition 38 Prozent und Rot-Rot-Grün nur 30 Prozent; Vorwahlerhebung im Rahmen der ARD-Wahlberichterstattung vom September 2009. 
litische Erwägungen im Vordergrund (+14 Punkte gegenüber 2005). Mit dieser eindeutigen Ausrichtung schafften es die Grünen, in dieser Domäne ihren Wettbewerbsvorsprung vor den anderen Parteien gegenüber 2005 sogar noch auszubauen: 64 Prozent der Wahlberechtigten schrieben ihnen hier die höchste Kompetenz zu (+8 Punkte), immerhin 44 Prozent darüber hinaus auch in der Energiepolitik. Zumindest der eigenen Wählerschaft gelten die Grünen außerdem als die Partei mit den besten bildungspolitischen Angeboten und der überzeugendsten Integrationspolitik. Ihr programmatischer Schwerpunkt liegt damit klarer als die Jahre zuvor auf der kulturellen Ebene. Die Grünen verstanden es aber auch, ihre Anhängerschaft über ihre sozialpolitischen Angebote anzusprechen, was vor allem enttäuschten SPD-Wählern den Wechsel erleichterte. Ihre stärker genderorientierte und auf einen Ausgleich der Generationen ausgerichtete Interpretation von sozialer Gerechtigkeit machte sie weiterhin für jüngere und weibliche Wähler besonders attraktiv. So führten Grünen-Wähler für ihre Wahlentscheidung abgesehen von ökologischen Gründen vor allem Aspekte der sozialen Gerechtigkeit an. Bildungspolitische Belange rangierten auf Platz drei und spielten damit eine stärkere Rolle als bei den Wählern anderer Parteien.

Das Spitzenpersonal ist traditionell bei der betont sachorientierten Wählerschaft der Grünen von untergeordneter Bedeutung. Es war aber gewiss kein Nachteil, dass das Spitzenduo Renate Künast und Jürgen Trittin nicht nur in der eigenen Anhängerschaft hohe Anerkennung genoss. Ihre Spitzenkandidaten trugen zu der hohen Authentizität der Partei bei: Jeder zweite Bundesbürger gesteht den Grünen eine hohe Glaubwürdigkeit ihrer Politik zu, womit sie deutlich vor den anderen Parteien, vor allem deutlich vor Union und SPD rangieren. Ihre Wähler fühlen sich den Grünen heute auch enger verbunden als die Wähler anderer Parteien. Solange sie den hohen moralischen Ansprüchen ihrer Anhänger gerecht werden, gibt ihnen das auch einen höheren Grad an Freiheit bei der Auswahl möglicher Koalitionspartner. Anders als in den Wahlen zuvor hatten die Grünen-Wähler keine klare Koalitionspräferenz. In der ARD-Wahltagsbefragung sprachen sich 39 Prozent für Rot-RotGrün, 32 Prozent für eine Ampelkoalition und immerhin knapp jeder Fünfte für eine Jamaika-Regierung oder für ein Zusammengehen mit der Union aus. Auch wenn sich die Mehrheit der Grünen politisch eher links einstuft, ist der Zusammenhalt zwischen SPD und Grünen auch auf der Wählerseite seit der Beendigung des rot-grünen Projektes erkennbar schwächer geworden. Ein deutliches Indiz dafür ist der erheblich verringerte Anteil von Grünen-Wählern, die ihre Erststimme dem SPD-Kandidaten gaben. Betrug der Anteil dieser grünen Splittingwähler bei der Bundestagswahl 2005 noch 58 Prozent, so waren es vier Jahre später nur noch 33 Prozent ${ }^{45}$.

\section{Linke-Wähler}

Ähnlich wie die Wähler der Grünen orientieren sich auch die Anhänger der Linken an einem dominanten Politikfeld: 61 Prozent gaben der Linkspartei vor allem wegen ihres Einsatzes für soziale Gerechtigkeit die Stimme. Für jeden Dritten spielte auch die Arbeitsmarktpolitik, hier vor allem höhere Löhne, eine entscheidende Rolle. Diese beiden Politikfelder bilden aus Sicht ihrer Anhänger den Kompetenzschwerpunkt der Linken. Darüber hinaus finden aber auch ihre bildungs- und rentenpolitischen Vorstellungen viel $\mathrm{Zu}$ -

45 Infratest dimap, a.a.O. (Fn. 19), S. 65. 
stimmung bei der eigenen Klientel. Dies gilt ebenso für ihre Forderung nach sofortigem Abzug aus Afghanistan, die ihr ein Alleinstellungsmerkmal sicherte und viel Zuspruch auch von Anhängern anderer Parteien brachte. Personelle Aspekte sind bei Wählern der Linken von eher untergeordneter Bedeutung, nur 12 Prozent von ihnen orientierten sich bei ihrer Entscheidung nach eigenen Aussagen an den beiden Spitzenkandidaten. Für die Mobilisierung der eigenen Wählerschaft dürfte aber das hohe Ansehen, das Gysi und Lafontaine in der eigenen Anhängerschaft genießen - mit Lafontaine waren 81 Prozent zufrieden, mit Gysi sogar 90 Prozent - förderlich gewesen sein. Die starke Ablehnung, die Lafontaine seitens der anderen Parteien erfuhr, war für den Erfolg der Linken nicht abträglich. Mit seiner kompromisslosen Haltung gegenüber Hartz IV, der Rente mit 67 oder dem Afghanistaneinsatz trug er maßgeblich zu deren Profilierung als linke Kraft bei, die sich am stärksten für die Belange der sozial Schwachen einsetzt. Nicht zuletzt deswegen stellte sie speziell im Westen für enttäuschte sozialdemokratische Wähler, vor allem für Arbeitslose und Angehörige des abgehängten Prekariats ${ }^{46}$, die attraktivere Alternative zur SPD dar. Wie tief die Enttäuschung gegenüber den Sozialdemokraten sitzt, lässt sich unter anderem daran ablesen, dass kaum ein Linksparteiwähler dem SPD-Kandidaten in seinem Wahlkreis die Erststimme gab, selbst wenn der Kandidat der Linken chancenlos war. Paradoxerweise plädierte aber gleichwohl die große Mehrheit (83 Prozent) für ein Zusammengehen mit der SPD und den Grünen. Dabei war nebensächlich, dass dafür kaum Chancen bestanden, denn das Votum der Linken-Wähler ist stärker als das aller anderen Wähler von Protest geprägt sowie davon, bestimmte Regierungen, wie etwa die Große Koalition, zu verhindern.

\section{Wähler sonstiger Parteien}

Von den Parteien, die den Sprung in den Bundestag verpassten, sollten die Piraten und die NPD nicht unerwähnt bleiben. Ihre Wähler weisen einige sozio-strukturelle Überschneidungen auf - sie sind vor allem jung und männlich -, ihre Motive könnten allerdings unterschiedlicher kaum sein. Die NPD wird nach wie vor in erster Linie wegen ihrer restriktiven Ausländerpolitik gewählt - ein Aspekt, der den Wählern der Piratenpartei völlig fern liegt. Sie lassen sich in ihrem Wahlverhalten von einem Bündel unterschiedlichster Motive leiten, wobei vor allem soziale Gerechtigkeit, die Bildungs- und die Rechtspolitik im Vordergrund stehen. Letzteres spielt zwar auch in der NPD-Wählerschaft eine gewisse Rolle, allerdings mit einer völlig anderen Konnotation. Anhänger der Piraten liegt in erster Linie die „Freiheit des Netzes“ am Herzen, NPD-Anhängern eine härtere Strafverfolgung vor allem von Ausländern. Unterschiedlich ist auch die Bindung an die Partei. Von den NPDWählern fühlt sich immerhin jeder fünfte der Partei eng verbunden und gibt an, sie immer zu wählen. Die Piraten verfügen als neu gegründete Partei naturgemäß noch über keinerlei Stammwählerschaft. Die politische Ausrichtung ihrer Anhängerschaft ist eher diffus, was vor allem an ihrem Splittingverhalten deutlich wird. Ihre Wähler waren zum Stimmensplitting mehr oder weniger gezwungen, weil für die Piratenpartei kaum Direktkandidaten antraten. Jeweils ein Fünftel von ihnen gab den Bewerbern der SPD oder der Grünen die Erststimme, jeder Vierte entschied sich für Kandidaten von Union oder FDP, jeder Sechste

46 Siehe dazu Rita Müller-Hilmer, Gesellschaft im Reformprozess, Studie der TNS Infratest Sozialforschung im Auftrag der Friedrich-Ebert-Stiftung, http://www.fes.de/inhalt/Dokumente/ 061017_Gesellschaft_im_Reformprozess_komplett.pdf (Abruf am 8. Februar 2010). 
für die der Linken und jeder Zehnte für Kandidaten anderer kleiner Parteien - mit Ausnahme der NPD. Der Offenheit der Piratenwähler steht eine relative Abschottung der NPD-Klientel gegenüber, deren Zweitstimmenwähler sich zu 80 Prozent auch mit der Erststimme für einen NPD-Kandidaten entschieden, auch wenn dieser naturgemäß keinerlei Chance auf ein Weiterkommen hatte.

\section{Nichtwähler}

Nichtwähler sind schwer auszumachen, denn in der Wahltagsbefragung werden sie nicht erfasst, und in den Vorwahlerhebungen geben sie sich nicht ohne weiteres zu erkennen oder bezeichnen sich häufig als noch unentschieden. Ihre Zahl lag bei dieser Wahl deutlich höher als vor vier Jahren. Noch in der Woche vor der Wahl zeigte sich jeder fünfte Wähler unentschlossen, wobei anzunehmen ist, dass der Großteil von ihnen der Wahl fernblieb. Nachfragen bei Nichtwählern ergaben, dass ein eher kleiner Teil prinzipiell politikfern ist und sich in keiner Weise für Politik interessiert (20 Prozent der Nichtwähler ${ }^{47}$ ). Häufiger verbreitet ist die Annahme, dass man mit seiner Stimmabgabe nichts bewirken könne (35 Prozent). Der Großteil derer, die am Wahltag zu Hause bleiben, begründet seine Wahlenthaltung aber mit Enttäuschungserfahrungen. Sieben von zehn geben an, sich früher immer für eine Partei entschieden zu haben, dass ihnen dies aber derzeit zu schwer falle. Ebenso groß ist der Anteil der Nichtwähler, die „den Politikern“ unterstellen, im Wesentlichen ihre eigenen Interessen zu verfolgen, oder beklagen, dass keine Partei mehr die Belange „der Normalbürger" vertritt. Wie schon während der gesamten Wahlperiode war die Verunsicherung unter SPD-Wählern deutlich höher als bei anderen Parteianhängern; so dass sie letztlich deutlich mehr Wähler an das Lager der Nichtwähler verlor als alle anderen Parteien zusammengenommen.

\section{Regierungsbildung und Oppositionsformierung}

Die Eindeutigkeit des Wahlergebnisses der Bundestagswahl 2009 erübrigte schon am frühen Wahlabend jegliche Diskussion über die künftige Zusammensetzung der Regierung. Bereits in den ersten Interviews nach Schließung der Wahllokale bekräftigten CDU, CSU und FDP ihr Wahlversprechen, gemeinsam eine Regierung zu bilden. Die anschließenden Koalitionsverhandlungen machten aber rasch deutlich, dass aufgrund der unterschiedlichen Ausgangssituation der drei Parteien eine Einigung nicht so leicht zu bewerkstelligen sein würde wie in den schwarz-gelben Regierungen früherer Jahre. Die erstarkte FDP wollte „kein Jota“ von ihren Wahlversprechungen abrücken und der neuen Regierung das Etikett einer „Regierung des Aufbruchs“ aufdrücken. Dies konnten die CDU und ihre Kanzlerin Merkel nicht zulassen, wäre es doch einem Dementi der aus ihrer Sicht recht erfolgreichen Regierungsarbeit der letzten vier Jahre gleichgekommen. Sie pochten vielmehr auf den Bestand der noch gemeinsam mit der SPD beschlossenen Maßnahmen etwa im Bereich der Bankenverstaatlichung und des Mindestlohnes - beides Gräuel für liberales Wirtschaftsverständnis. Die von den bayerischen Wählern arg gebeutelte CSU sah sich veranlasst, in den

47 Die folgenden Befunde zu den Nichtwählern beruhen auf kumulierten Ergebnissen der ARDDeutschlandTrends von Infratest dimap vor der Bundestagswahl 2009. 
Koalitionsverhandlungen wieder verstärkt als die Stimme des kleinen Mannes aufzutreten. So einig sie sich dabei mit den Liberalen in der Frage der Steuersenkung war, so groß war der Dissens in Sachen Gesundheitsreform. Bemerkenswert geräuschlos wurden nur die Streitpunkte in der Innen- und Rechtspolitik beiseite geräumt. Am Ende der Verhandlungen verkündete Westerwelle auf einem außerordentlichen Parteitag der Liberalen: „Alle zwanzig Kernvorschläge der FDP, alle, konnten in der Koalitionsvereinbarung umgesetzt werden“ 48 - eine Wertung, die bei der Union nicht gerade Freude auslöste.

Insgesamt vermittelte die Koalition von Beginn an den Eindruck von Uneinigkeit nicht nur in Einzelpunkten, sondern auch in der Linienführung. Am deutlichsten wurde der Dissens in dem Zielkonflikt zwischen Steuersenkung einerseits und dem Abbau der Staatsschulden andererseits - beides von den Koalitionspartnern beschlossen. Im Koalitionsvertrag wurde dieser Widerspruch mit dem Versprechen auf wachsende Einnahmen eher in die Zukunft verlagert als gelöst, weshalb sich das gesamte Kabinett wenige Wochen nach der Regierungsbildung schon zur ersten Klausur im brandenburgischen Meseberg einfand, um unterschiedliche Ausdeutungen des Koalitionsvertrages und drohende Konflikte aufzuarbeiten. Nachdem auch dies nicht zur vollständigen Klärung der grundlegenden Konflikte zwischen den drei Koalitionsparteien beitrug, war schnell von einem „schwarz-gelben Fehlstart“ 49 die Rede oder von einer „bürgerliche Koalition“, die nichts sei „als ein Sehnsuchtsort, den sich Konservative und Liberale in zehn Jahren Rot-Grün und großer Koalition zusammengeträumt haben "50. Aber nicht nur Medienbeobachter rieben sich verwundert die Augen; auch innerhalb der Koalition wurden immer mehr kritische Stimmen laut. So entdeckte Bundestagspräsident Norbert Lammert in dem „Wachstumsbeschleunigungsgesetz“ - Kernstück des Koalitionsvertrages - „manche zweifelhafte und einige...schlicht misslungene und auch nicht vertretbare Regelungen"51. Die FAZ zog zum Jahresende folgendes Fazit: „Nie zuvor haben sich die Partner einer neu gebildeten Koalition so schnell auf dermaßen vielen Politikfeldern dermaßen heftige Auseinandersetzungen geliefert. " 52 Nach einem Wahlergebnis der Superlative folgte ein Fehlstart der Superlative.

Vergleichsweise unproblematisch verlief die Verteilung der Ministerien zwischen den drei Partnern. Die CDU stellt neben der Bundeskanzlerin weitere sieben Ministerien, darunter die strategisch bedeutsamen Häuser Finanzen und Inneres sowie die gesellschaftspolitisch wichtigen Ministerien Arbeit und Soziales, Bildung und Familie. Ihre Schwesterpartei musste zwar das prestigeträchtige Wirtschaftsministerium abgeben, bekam dafür aber mit dem Verteidigungs-, dem Landwirtschafts- und Verbraucher- sowie dem Verkehrsministerium ungeachtet ihrer Stimmenverluste drei statt bisher zwei Ministerien zugewiesen. Die FDP erhielt neben dem Außenamt die Ministerien Wirtschaft, Justiz, Entwicklungshilfe und Gesundheit, wo sie sich gegen die CDU durchsetzte. ${ }^{53}$ Nach nur vier Wochen war Merkel zu einer ersten Umbesetzung gezwungen. Aufgrund neuer Informationen zu dem Militäreinsatz in Kundus wenige Wochen vor der Wahl musste der vom Verteidigungsmi-

48 Peter Carstens / Günter Bannas, Westerwelle: Wir haben uns in allem durchgesetzt, in: FAZ vom 26. November 2009, S. 1.

49 Jan Dams, Schwarz-gelber Fehlstart, in: Die Welt vom 3. Dezember 2009, S. 3.

50 Brigitte Fehrle, Am Anfang das Ende, in: Berliner Zeitung vom 28. November 2009, S. 2.

51 Günter Bannas, Lammert kritisiert die Koalition, in: FAZ vom 28. Dezember 2009, S. 4

52 Ders., Nur Jamaika wäre noch zerstrittener, in: FAZ vom 8. Januar 2010, S. 8

53 Zur Regierungsbildung nach der Bundestagswahl 2009 siehe ausführlich den Beitrag von Thomas Saalfeld in diesem Heft der ZParl. 
nister zum Arbeitsminister gewechselte Franz Josef Jung zurücktreten - der schnellste Rücktritt eines Bundesministers in der Geschichte der Bundesrepublik. Für ihn wechselte Ursula von der Leyen ins Arbeitsministerium, und die bis dato wenig bekannte CDU-Bundestagsabgeordnete Kristina Schröder (vormals Köhler) übernahm das Familienministerium.

Die Kabinettsbildung erzwang weitere Umbesetzungen an der Spitze der drei Parteien. Herrmann Gröhe folgte Ronald Pofalla im Amt des CDU-Generalsekretärs, neuer CSULandesgruppenchef wurde Hans-Peter Friedrich, neuer Generalsekretär der FDP Christian Lindner.

Tiefgreifende Personaländerungen waren auch bei der SPD nach der verheerenden Niederlage unumgänglich. Schon kurz nach der Wahl kündigte Müntefering an, die Verantwortung für die Stimmenverluste zu übernehmen und zurückzutreten. Ihm tat es kurz darauf der Generalsekretär Hubertus Heil gleich. Da zuvor schon klar war, dass Peter Struck aus dem Bundestag ausscheiden würde, war auch der dritte Spitzenposten der SPD vakant. Noch am Wahlabend machte Steinmeier seinen Anspruch auf das Amt des Fraktionsvorsitzenden deutlich, schloss aber gleichzeitig die Übernahme des Parteivorsitzes aus. Im kleinen Kreise verständigten sich führende SPD-Politiker darauf, dass Siegmar Gabriel für den Parteivorsitz und Andrea Nahles als Generalsekretärin auf dem Dresdner Parteitag im November kandidieren würden. Dort kam es zu einer ebenso grundsätzlichen wie emotionalen kritischen Aussprache über die zurückliegenden elf Regierungsjahre der SPD, die Gabriel in seiner Bewerbungsrede aufgriff und die SPD zur Erneuerung aufforderte. Die Bereitschaft der Partei, ihm dabei zu folgen, fand ihren Ausdruck in einem unerwartet hohen Wahlergebnis für Gabriel als neuen Parteivorsitzenden. Auch die von ihm vorgeschlagenen vier Stellvertreter, die Vorsitzende aus Nordrhein-Westfalen, Hannelore Kraft, Manuela Schwesig, Sozialministerin in Mecklenburg-Vorpommern, Ex-Arbeitsminister Olaf Scholz sowie der Regierende Bürgermeister Berlins, Klaus Wowereit, wurden mit jeweils großer Mehrheit gewählt. Die ebenfalls zur neuen Generalsekretärin gewählte Andrea Nables musste allerdings einige Neinstimmen verkraften.

Ohne Überraschungen verlief die Wahl der Fraktionsvorsitzenden der Grünen, bei denen Renate Künast im Amt bestätigt und Jürgen Trittin ihr neu zur Seite gestellt wurde. Größere Verwerfungen deuteten sich aber schon früh bei der Linkspartei an. Lafontaine kündigte auf einer Fraktionsklausur völlig überraschend seinen Rücktritt als Co-Vorsitzender der Bundestagsfraktion an und regte gleichzeitig an, sowohl dieses Amt als auch den Parteivorsitz - nach dem angekündigten Rückzug Lothar Biskys - mit einer Doppelspitze zu besetzen: jeweils eine Frau und ein Mann, davon eine(r) aus dem Westen und eine(r) aus dem Osten. Kurz darauf wurde zudem bekannt, dass er sich einer Krebsoperation unterziehen müsse und deshalb unklar sei, ob er gesundheitlich in der Lage sein würde, das Amt des Bundesvorsitzenden weiterhin wahrzunehmen. Daraufhin entbrannten eine Nachfolgediskussion und ein neuerlicher Ost-West-Konflikt. Angesichts der Bedeutung Lafontaines für die Zusammenführung von PDS und WASG zur Linkspartei und für deren anschließenden Wahlsieg erscheint die Zukunft der Linkspartei deutlich ungewisser als noch vor kurzem erkennbar.

Einige dieser Ereignisse sind nicht zuletzt Ausfluss der durch die Bundestagswahl 2009 ausgelösten tiefgreifenden Veränderung des Parteiengefüges. Offen bleibt, welche mittelund langfristigen Auswirkungen sie auf das Kräfteverhältnis zwischen den Volksparteien und den „kleinen“ Parteien haben werden, zwischen den beiden Regierungspartnern und zwischen SPD und Linkspartei. Klar ist nur, dass auch nach der Wahl vieles im Fluss bleibt. 


\section{Zusammenfassung und Ausblick}

Die Bundestagswahl 2009 hat nicht nur zu einem Regierungswechsel geführt, sondern hat vor allem ein völlig verändertes politisches Kräfteverhältnis zwischen den Parteien zur Folge, ohne dass sich jedoch die politische Landschaft grundlegend gewandelt hätte. Das nach der Wiedervereinigung entstandene Fünf-Parteien-System geht konsolidiert aus dieser Wahl hervor, mit allerdings geschwächten Volksparteien und erstarkten „kleinen“ Parteien. Dass auch in Zeiten einer Großen Koalition keine weitere Partei den Sprung ins Parlament schaffte, kann als Zeichen der Stärke und Flexibilität des neu austarierten Parteiensystems gewertet werden. Der deutlich gestiegene Nichtwähleranteil eröffnet neuen Wettbewerbern aber auch neue Chancen, wie das Beispiel der Piratenpartei zeigt, die aus dem Stand zwei Prozent erzielte. Die Wahlenthaltung vor allem vieler enttäuschter SPD-Wähler trug aber auch dazu bei, dass am Ende wieder eine klassische, lagerkonforme Zweierkoalition eine stabile Mehrheit bekam.

Das Wahlergebnis spiegelt eine neue Stufe der Volatilität im Wahlverhalten wider. Parteiwechsel sind nicht beliebig, aber aufgrund abnehmender Parteibindungen, stärkerer Ausdifferenzierung von Interessen und der Herausbildung neuer Konfliktlinien immer unberechenbarer, nicht zuletzt deshalb, weil auch strategische und koalitionsbezogene Überlegungen immer wichtiger werden.

Politisch steht das Ergebnis der Bundestagswahl 2009 für die Hoffnung auf wirtschaftliche Stabilität und Erhalt der internationalen Wettbewerbsfähigkeit Deutschlands. Dies versprachen sich die Wähler in der Krise eher von einer schwarz-gelben Regierung unter der Führung von Angela Merkel. Die vormals starken Vorbehalte gegenüber einer CDU/CSUFDP-Regierung vermochte vor allem die Kanzlerin erfolgreich zu entkräften. Die entscheidenden Vorteile von Schwarz-Gelb gegenüber anderen Konstellationen waren eine klare strategische und programmatische Positionierung beider Partner, klare Führungsstrukturen und vor allem der auf beiden Seiten deutlich erkennbare Wille, gemeinsam zu regieren.

Die SPD hatte dem wenig entgegenzusetzen. Sie zog kaum Lehren aus der Wahlniederlage 2005 und verstand es nicht, sich innerhalb der Großen Koalition neu zu positionieren. Die vielen Wechsel an der Parteispitze trugen eher zur Verwirrung als zur Neuprofilierung bei. Letztlich konnte sie große Teile ihrer angestammten Wähler weder programmatisch noch von der angebotenen Machtperspektive überzeugen. Am Ende verlor sie Wähler nach rechts wie nach links sowie in noch größerer Zahl an die Nichtwähler. Mit einem Stimmenanteil von 23 Prozent erscheint ihr Status als Volkspartei gefährdet.

Linke und Grüne profitierten am meisten von der Schwäche der SPD, zumal es ihnen gelang, ihre jeweiligen Kernkompetenzen (Linke: Soziale Gerechtigkeit / Grüne: Ökologie) in der Opposition zu stärken. Beide konnten infolgedessen deutlich an Stimmen und Mandaten zulegen. Die Grünen behielten dabei im Wesentlichen das für sie typische Wählerprofil bei (höher gebildete, städtische Wähler jüngeren bis mittleren Alters). Die Linke konnte sich unter Führung von Lafontaine in den westlichen Bundesländern etablieren, wo sie vor allem von Arbeitern und Arbeitslosen gewählt wurde, anders als in Ostdeutschland aber noch weit entfernt ist vom Status einer in allen Bevölkerungsschichten vertretenen Volkspartei.

Gleichwohl geht mit der Erstarkung der Linken im Westen eine gewisse Angleichung regionaler Wahlmuster einher. Diese wird noch dadurch verstärkt, dass die CDU im Osten - seit Jahren ihre Problemregion - zulegte bei gleichzeitig starken Verlusten in ihren tradi- 
tionellen Hochburgen im Süden. Auch die FDP und die Grünen konnten bei deutlichen Gewinnen in den neuen Bundesländern ihre Westlastigkeit etwas abbauen. In der regionalen Betrachtung wird die Schwächung der SPD besonders deutlich, die sich nur in einem Bundesland als stärkste Kraft behaupten konnte - 2005 waren es noch zwölf. Im Osten verlor sie ihre Spitzenposition und fiel hinter die Linke und die CDU auf den dritten Platz zurück. Auch in vielen Städten wurde sie nur noch drittstärkste Kraft. Die Anzahl direkt gewonnener Mandate reduzierte sich von 150 auf 64.

Die neue Regierung geht im Vergleich zur letzten schwarz-gelben Koalition mit einem völlig neuen Kräfteverhältnis der drei Partner und mit einer veränderten Rollenverteilung an den Start. Die modernisierte CDU hat zwar an wirtschafts- und steuerpolitischem Profil verloren, steht aber für eine neue Familien- und Klimapolitik. Gemeinsam mit der CSU gilt sie zudem ihrer Wählerschaft als Garant für eine konservative Rechts- und Innenpolitik sowie für die Sicherung sozialer Mindeststandards. Der bundespolitische Part der CSU besitzt darüber hinaus noch keine klaren Konturen, wohl aber personelle Perspektiven in der Person zu Guttenbergs. Am nachhaltigsten gewandelt hat sich die Situation der FDP, die immerhin halb so stark ist wie die CDU und mehr als doppelt so viele Stimmen einbringt wie die CSU. Ihre deutlich gestärkte Position verdankt sie in erster Linie ihrer konsequenten marktwirtschaftlichen und ordnungspolitischen Orientierung und der Erwartung vieler „Leistungsträger“, mehr vom erhofften Aufschwung zu profitieren.

In der neuen Regierung zeichneten sich schon früh Konflikte ab zwischen einer qua Wählerauftrag zu grundlegenden Reformen verpflichteten FDP und der pragmatisch ausgerichteten, Ideologien gegenüber heute eher zurückhaltenden Union. Letztere wird zudem nichts unversucht lassen, um wieder an wirtschaftspolitischem Profil zu gewinnen und zu alter Stärke zu finden.

Durch die Kräfteverschiebungen im linken Parteienspektrum zeichnet sich auch in der Opposition eine neue Rollenverteilung ab. Als 23-Prozent-Partei stellt die SPD nicht mehr das unumstrittene Zentrum im linken Lager dar. Nach elf Jahren Regierungsbeteiligung muss sie sich in der Opposition im Spannungsfeld zwischen Freiheit und sozialer Gerechtigkeit neu positionieren, begegnet auf diesen Feldern mit den Grünen beziehungsweise der Linken aber jeweils erstarkten und selbstbewussten Kontrahenten ${ }^{54}$.

Die Wähler haben mit ihrem volatilen Verhalten eine ganz neue politische Konstellation geschaffen, die nur auf den ersten Blick der Stabilität früherer Tage gleicht. Sie haben das Konzept dominierender Volksparteien in Frage gestellt und den „kleinen“ Parteien völlig neue Spielräume für Koalitionsalternativen eröffnet - lagerübergreifende Bündnisse inbegriffen. Wer auch bei Gefahr partieller Wählerverluste die Fünf-Prozent-Hürde nicht wie früher fürchten muss, kann wagemutiger sein, so wie es die Grünen schon seit einiger Zeit vormachen. Sollten sich diese neuen Konstellationen als erfolgreich erweisen, wird dies nicht ohne Auswirkungen auf die Positionierung der anderen Parteien und auf künftiges Wahlverhalten bleiben. Es ist deshalb davon auszugehen, dass der Wählermarkt in den nächsten Jahren in Bewegung bleibt.

54 Dieses neue Selbstbewusstsein äußert sich beispielsweise in dem Anspruch, auf Landesebene den Regierungschef zu stellen, wie bei der Linken zuletzt in Thüringen und bei den Grünen perspektivisch in Berlin. 